Article

\title{
New Digital Plug and Imaging Sensor for a Proton Therapy Monitoring System Based on Positron Emission Tomography
}

\author{
Nicola D'Ascenzo ${ }^{1,2,3, *}$, Min Gao ${ }^{1}$, Emanuele Antonecchia ${ }^{1,3}$, Paolo Gnudi ${ }^{3,4}$, \\ Hsien-Hsin Chen ${ }^{5,6}$, Fang-Hsin Chen ${ }^{5}{ }^{-}$, Ji-Hong Hong ${ }^{6,8} \oplus^{-}$, Ing-Tsung Hsiao ${ }^{5}$, \\ Tzu-Chen Yen ${ }^{7}$, Weidong Wang ${ }^{9}$, Daoming $\mathrm{Xi}^{10}{ }^{10}$, Bo Zhang ${ }^{11}$ and Qingguo Xie ${ }^{1,2,3, *}$
}

1 School of life science and technology, Huazhong University of Science and Technology, Wuhan 430074, China; april_mingao1@hust.edu.cn (M.G.); emanuele.antonecchia@neuromed.it (E.A.)

2 Wuhan National Laboratory of Optoelectronics, Wuhan 430074, China

3 Istituto Neurologico Mediterraneo, NEUROMED IRCCS, Pozzilli 86077, Italy;

Paolo.Gnudi@student.hs-rm.de

4 Department of Biomedical Engineering, RheinMain University of Applied Sciences, Wiesbaden 65203, Germany

5 Department of Medical Imaging and Radiological Science, Chang Gung University, Taoyuan 33302, China; hsin0426@gmail.com (H.-H.C.); fanghsinchen@mail.cgu.edu.tw (F.-H.C.); ihsiao@mail.cgu.edu.tw (I.-T.H.)

6 Department of Radiation Oncology, Chang Gung Memorial Hospital at Linkou (CGMH at Linkou), Taoyuan 33302, China; jihong@cgmh.org.tw

7 Molecular Imaging Center and Nuclear Medicine, Chang Gung Memorial Hospital/Chang Gung University (CGMH/CGU), Taoyuan 33302, China; yen1110@cloud.cgmh.org.tw

8 Institute for Radiological Research, Chang Gung Memorial Hospital/Chang Gung University (CGMH/CGU), Taoyuan 33302, China

9 Key Laboratory of Biomedical Engineering and Translational Medicine, Ministry of Industry and Information Technology, Beijing 100191, China; wangwd301@126.com

10 Raycan Technology Co., Ltd., Suzhou 215163, China; daoming.xi@raymeasure.com

11 RaySolution Digital Medical Imaging Co., Ltd., Ezhou 436044, China; bo.zhang@digital-pet.com

* $\quad$ Correspondence: ndasc@hust.edu.cn (N.D.); qgxie@hust.edu.cn (Q.X.); Tel.: +86-027-8998-1598 (N.D. \& Q.X.)

Received: 27 July 2018; Accepted: 2 September 2018; Published: 8 September 2018

\begin{abstract}
One of the most challenging areas of sensor development for nuclear medicine is the design of proton therapy monitoring systems. Sensors are operated in a high detection rate regime in beam-on conditions. We realized a prototype of a monitoring system for proton therapy based on the technique of positron emission tomography. We used the Plug and Imaging (P\&I) technology in this application. This sensing system includes LYSO/silicon photomultiplier (SiPM) detection elements, fast digital multi voltage threshold (MVT) readout electronics and dedicated image reconstruction algorithms. In this paper, we show that the P\&I sensor system has a uniform response and is controllable in the experimental conditions of the proton therapy room. The prototype of PET monitoring device based on the P\&I sensor system has an intrinsic experimental spatial resolution of approximately $3 \mathrm{~mm}$ (FWHM), obtained operating the prototype both during the beam irradiation and right after it. The count-rate performance of the P\&I sensor approaches 5 Mcps and allows the collection of relevant statistics for the nuclide analysis. The measurement of both the half life and the relative abundance of the positron emitters generated in the target volume through irradiation of $10^{10}$ protons in approximately $15 \mathrm{~s}$ is performed with $0.5 \%$ and $5 \%$ accuracy, respectively.
\end{abstract}

Keywords: silicon sensors; digital readout; multi voltage threshold; positron emission tomography; proton therapy 


\section{Introduction}

Optical sensing imaging systems in nuclear medicine are based on the principle of conversion of the energy of gamma rays into visible light in scintillation materials. Optical sensors are used to read out the scintillation light and to convert it into an electrical signal for further analysis. The typical wavelength sensitivity of modern sensors in this application should peak between $400 \mathrm{~nm}$ and $420 \mathrm{~nm}$ because the relevant scintillators emit light in this wavelength range. Monitoring systems for the dose delivered during proton therapy treatments are among the most critical applications in nuclear medicine. Recent advances in this area require in fact to design and test new image sensor systems with high rate detection capability in the wavelength range mentioned above. The development of dedicated digital readout electronics, possibly integrated within the sensor, represents a key issue in this respect $[1,2]$.

Positron emission tomography (PET) has been demonstrated to be the most efficient technique to control the delivered dose during proton therapy treatment in the 1990s [3-6]. Building on the study of the distribution of the positron-emitting nuclei generated by relativistic ion beams in biological matter, the first PET systems were installed in proton therapy treatment rooms. Since then, PET is dominating the panorama of modern research in the field of novel sensor development for frontier applications in nuclear medicine and radiation therapy, with the unique synergy of a large number of groups [7-27]. During a proton therapy treatment, cancer cells are bombarded with protons at energies typically ranging between $70 \mathrm{MeV}$ and $250 \mathrm{MeV}$. As ions are entirely stopped in the target volume, a reliable monitoring of the delivered dose can rely only on the detection of secondary radiation. PET-based proton therapy monitoring systems target the non-elastic nuclear interactions of protons with the target volume. Positron emitters as ${ }^{11} \mathrm{C},{ }^{10} \mathrm{C},{ }^{15} \mathrm{O},{ }^{13} \mathrm{~N},{ }^{12} \mathrm{~N}$ and ${ }^{8} \mathrm{~B}$ are generated with abundance ranging between $0.1 \%$ and $70 \%$. The time-correlated $511 \mathrm{keV}$ photon pairs produced in the annihilation of the emitted positrons are detected. Tomographic image reconstruction techniques are applied to the data to retrieve the space distribution of the positron emitters within the target volume during and after proton irradiation.

Information about the precision of the treatment is obtained by comparing the measured positron distribution in the target volume with a prediction based on the treatment plan and on the time course of the irradiation [28]. Recent improvements in the physical modeling of nuclear interactions in biological matter allow monitoring the maximum ion range, verifying the field position and quantifying the unpredictable deviations between prescribed and applied dose due to biological and anatomical changes in the patient [29].

Optical sensing for PET imaging plays a fundamental role in the design of highly accurate monitoring systems for proton therapy. Scintillating crystals read out by photomultiplier tubes-related technology constitute a traditional sensor solution for the detection of $511 \mathrm{keV}$ photons. By way of example, a prototype of dual panel PET detector with area $15 \times 15 \mathrm{~cm}^{2}$ was recently built based on a LYSO crystal matrix with $2 \mathrm{~mm}$ pitch read out by a position sensitive photomultiplier (H8500; HAMAMATSU) [30,31]. It was demonstrated that the presence of materials with different oxygen content can be detected with this technique. Although such technology allows a 1:1 coupling between crystals and photodetectors and is therefore suitable to the requirements of PET monitoring systems, it needs a bias voltage up to $1.5 \mathrm{kV}$ and constrains the design to blocks with minimal size of approximately $5 \times 5 \mathrm{~cm}^{2}$.

Besides traditional photodetectors, one of the most suitable sensors for this application is the silicon photomultiplier (SiPM). It is a low photon flux silicon sensor with an area ranging typically between $1 \mathrm{~mm}^{2}$ and $9 \mathrm{~mm}^{2}$ and an internal gain of $10^{6}$ reached at a bias ranging between $20 \mathrm{~V}$ and $60 \mathrm{~V}$. Modern commercially available SiPMs exhibit photon detection efficiency up to $40 \%$ at $420 \mathrm{~nm}$ and single photon time resolution less than 100 ps (FWHM) [32-34]. Radiation hardness is currently under investigation [35]. Due to their compact size and simple required readout electronics, they are substituting the traditional photomultiplier tubes in PET instrumentation for proton therapy monitoring systems. LYSO crystals with size $3.8 \times 3.8 \times 22 \mathrm{~mm}^{3}$ and read out by SiPM (dSIPM; 
PHILIPS) were used to build a dual panel PET detector with area $6.5 \times 6.5 \mathrm{~cm}^{2}$ [36]. To reduce the dark count rate (DCR) of the sensors, the system was kept at a temperature of $3{ }^{\circ} \mathrm{C}$. The modules were placed in separate Styrofoam boxes, which were continuously flushed with dry nitrogen gas to prevent condensation. The total DCR was further reduced significantly by disabling the top $20 \%$ of the microcells that exhibited the highest DCR. This prototype allowed a measurement of the fast decay of ${ }^{12} \mathrm{~N}$, which has an half-life of just $11 \mathrm{~ms}$, during the proton irradiation, increasing the rejection of the prompt photon background and consequently improving the image quality. SiPMs (MPPC; HAMAMATSU) are also currently used in the prototype of the INSIDE PET scanner, for the readout of Lutetium Fine Silicate (LFS) crystals with size $3 \times 3 \times 20 \mathrm{~mm}^{3}$ composing the $10 \times 25 \mathrm{~cm}^{2}$ PET panels [37,38].

As it follows from the above-mentioned detector concepts, the SiPM enabled to reach a new frontier in the sensor development for PET monitoring systems for proton therapy, namely the in-beam image acquisition. PET detectors need to be integrated with the proton delivery equipment and the signal registration is performed either during irradiation or immediately after it without moving the patient. The in-beam signal registration is beneficial to increment the collected signal statistics and to reduce the effect of biological washout, with a consequent improvement of the image quality and accuracy. The key issue is here the high level of background due to the prompt photons generated in nuclear interactions during proton irradiation. Several solutions are being tested, based on the background rejection using the spill time information [39]. Besides PET, other approaches to range determination using the prompt photons information were also developed [40-50].

In recent years, we have witnessed an improvement both in the development of dedicated SiPM technology based on the complementary metal oxide semiconductor (CMOS) process $[51,52]$ and in the realization of related high bandwidth digital readout electronics for PET [53-63]. As a possible solution to the sampling-rate challenge, we have previously proposed a multi voltage threshold (MVT) sampling method that takes samples of a pulse with respect to a set of reference voltages. By choosing the reference voltages properly, the MVT method can always obtain samples at the fast leading edge of a pulse. The total energy and the timing of the pulse can be obtained by the digital signal processing of a small set of samples of the pulse. We have shown that for PET detectors it is sufficient to use only a few reference voltages to obtain a small number of samples. As the pulses are instantaneously digitized and the number of samples per pulse is small, this sampling method can have a high count-rate capability without requiring a large on-board storage buffer $[64,65]$. This fast digital electronics is used to read out dedicated sensors based on the LYSO/SiPM coupling. The sensors are assembled in a compact configuration, which enables the design of PET systems with geometries adaptable to specific organs or clinical problems. A dedicated geometry-independent image reconstruction framework has been developed in order to provide a fast and effective analysis of the data collected by this sensor system. The combination of novel LYSO/SiPM sensor technology, MVT dedicated digital electronics and image reconstruction constitutes the Plug and Imaging (P\&I) sensor. We showed that, in optimal conditions, this imaging system can reach a crystal-level energy resolution of approximately $10 \%$ and a detector-level coincidence time resolution of 390 ps FWHM [56]. The system can support a maximal count rate of approximately 5 Mcps [62], being thus competitive with similar sensor and readout technologies for PET.

In the technological context outlined above, the P\&I sensor system would satisfy the demanding requirements of proton therapy monitoring systems. However, while we have already demonstrated its possible application to both preclinical and clinical standard PET devices, its feasibility for non-conventional systems has not been investigated yet. In this paper, we show, for the first time to our knowledge, the application of the P\&I sensor system to the more challenging scenario of proton therapy monitoring with PET. We built a prototype PET monitoring system on the basis of the P\&I sensor modules and we installed it at the proton delivery facility of the Chang Gung Memorial Hospital. In this paper, we show that the P\&I sensor system composed of LYSO/SiPM sensors with MVT dedicated digital readout and reconstruction has a uniform response and is controllable in the 
experimental conditions of the proton therapy room. The prototype of PET monitoring device based on the P\&I sensor system has an intrinsic experimental spatial resolution of approximately $3 \mathrm{~mm}$ (FWHM). A key-feature of this paper is that the count rate performance of the P\&I sensor approaches 5 Mcps and is higher than most of the reported prototypes of proton beam monitoring systems with digital readout. It allows both the beam-on operation and the collection of relevant statistics for a nuclide analysis in the beam-off time. We measure both the half life and relative abundance of the positron emitters generated in the target volume through the irradiation of $10^{10}$ protons in approximately $15 \mathrm{~s}$ with respective accuracies of $0.5 \%$ and $5 \%$.

The paper is structured as follows. In Section 2, we present the P\&I sensor technology, the PET monitoring system prototype and the research methods and purpose; in Section 3, we outline the results divided into measured technological parameters of the optical sensing system and prototype performance; and, finally, in Section 4, we discuss the results and perform a comparison with recent optical sensing systems developed for PET proton therapy monitoring devices.

\section{Materials and Methods}

\subsection{The Plug and Imaging Sensor System}

The basic module of the P\&I imaging sensor system is shown in Figure 1. It is composed of a $6 \times 6$ LYSO array read out by a $6 \times 6$ SiPM array (FM30035; SENSL, Cork, Ireland) and a printed circuit board (PCB) with the SiPM biasing circuit. The used SiPM is composed of 4774 microcells with a pitch of $35 \mu \mathrm{m}$ and a filling factor of $64 \%$ on a total area of $3 \times 3 \mathrm{~mm}^{2}$. It exhibits a $20 \%$ peak photon detection efficiency at the wavelength of $500 \mathrm{~nm}$. The average breakdown voltage is $27.5 \mathrm{~V}$, with a temperature dependence of $20 \mathrm{mV} / \mathrm{K}$. The LYSO matrix (JTC, Suzhou, China) contains $3.9 \times 3.9 \times 20 \mathrm{~mm}^{3}$ pixellated crystals with $0.3 \mathrm{~mm}$ crystal gaps that are filled with barium sulfate to reflect the scintillation light and isolate each crystal optically. Each SiPM has an active detection area of $3 \times 3 \mathrm{~mm}^{2}$. The SiPM and LYSO matrices, both having a $4.2 \times 4.2 \mathrm{~mm}$ pitch, are carefully aligned and coupled using epoxy optical adhesive (China Bluestar Chengrand Co., Ltd., Chengdu, China, Model: GHJ-01(Z) , refractive index 1.56 , thickness approximately $0.1 \mathrm{~mm}$, light transmittance $>90 \%$ for visible wavelengths, operation temperature $-60{ }^{\circ} \mathrm{C}-80{ }^{\circ} \mathrm{C}$ ). LYSO/SiPM elements are read out independently. The overall detection area of the module is approximately $25 \times 25 \mathrm{~mm}^{2}$.

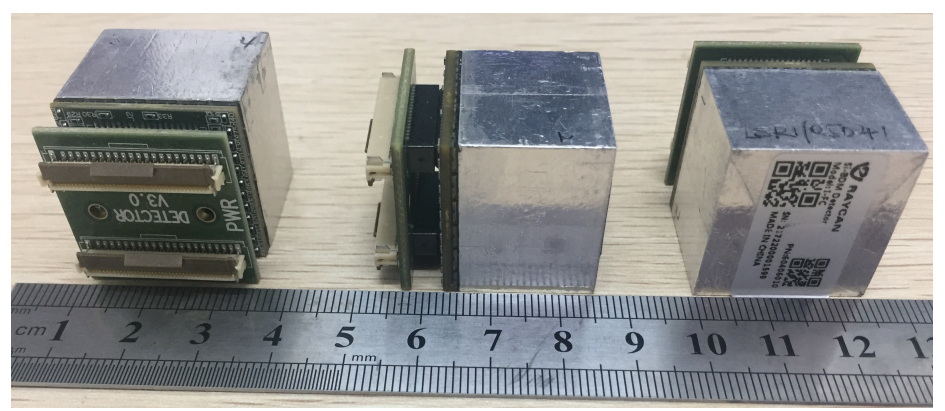

Figure 1. The P\&I sensor system. A $6 \times 6$ array of LYSO crystals with size $3.9 \times 3.9 \times 20 \mathrm{~mm}^{3}$ is read out by an array of $6 \times 6 \mathrm{SiPM}$. The assembled block has a dedicated plug-in system for the readout electronics on the back.

The digital readout electronics is shown in Figure 2. One electronic board serves two P\&I sensor modules, providing thus the biasing and readout to 72 independent SiPM/LYSO channels. Concerning the biasing, due to the intrinsic wafer non-uniformities in the production of the SiPMs, the sensors do not have the same breakdown voltage. The SiPMs used in the prototype have an average breakdown voltage of $24.5 \mathrm{~V}$, with a variation of $8 \%$ (FWHM). We grouped them so that each pair of P\&I modules exhibits a variation of approximately $2 \%$ (FWHM). An average overvoltage of $5 \mathrm{~V}$ is provided to each pair of modules by a board. Concerning the readout, as shown in Figure 3, we designed 
a two-channel FPGA-only MVT-digitizer board by using an Altera EP4CE115F29I7N FPGA, with 114,480 logic elements (LE), 3888 Kbits embedded memory, 266 embedded $18 \times 18$ multipliers, 4 general-purpose PLLs, 20 Global Clock Networks, 8 User I/O banks, and 528 maximum user I/O. A 24-Channel 4-level MVT digitizer is using 51\% LE and 46\% I/O of the FPGA chip. We are currently developing a 36-ch 4-levels MVT digitizer using the same chip. Each MVT channel has four comparators to provide four reference voltages. A key feature of the implementation is to use the differential I/Os of the FPGA working in the low voltage differential signaling (LVDS) receiver mode to function as voltage comparators (they are referred to as the LVDS comparators below). A PET event pulse generated by a LYSO/SiPM sensor is split into four signals, each of which is sent to an LVDS comparator. The logic output of the LVDS comparator is connected to two TDCs for determining the digital times of its positive and negative transitions, therefore generating a total of eight samples for a pulse with sufficient amplitude. Since the voltage values are known a priori, these samples need to contain the time values generated by the TDCs. The eight time samples generated for a pulse are packaged into an event-word and stored in a FIFO. The FIFO data are sent out for analysis via an Ethernet interface. The LVDS comparators, TDCs, event identify and pack module, and FIFO are all implemented on the FPGA. In addition, a data send module for talking to an Ethernet interface is implemented in the FPGA. The TDC is governed by a $200 \mathrm{MHz}$ clock and the counter (the digital coarse time) has 40 bits, capable of counting up to about $91.7 \mathrm{~min}$ at 5 -ns increment. In other words, the time stamp of the TDC is composed of two parts, a coarse time and a fine time. The coarse time is obtained with the counter and has a resolution of $5 \mathrm{~ns}$, the fine part is limited by the 90 ps minimal bin width of the TDC. The reference voltages are provided using Texas Instrument (TI) DAC7678. All MVT channels are synchronized to an external sync clock. With respect to the previous implementation [56], a $1 \mathrm{G}$ Ethernet interface is used. Each threshold produces two 48-bit time samples that are packed into a 192-bit record. Therefore, a maximal count rate of 5 Mcps is achievable. The design is compatible with a $10 \mathrm{G}$ Ethernet interface, which would further improve the maximal count rate allowed.

The intrinsic properties of the LYSO/SiPM sensor impose a physical limitation to the count rate per channel. As the decay time of the LYSO crystal is approximately $40 \mathrm{~ns}$, the whole generated signal has a duration of less than 200 ns. By using a paralizable dead-time model with a 200-ns dead time, we have $<10 \%$ dead-time loss at event rates below approximately $160 \mathrm{kcps}$ per channel.

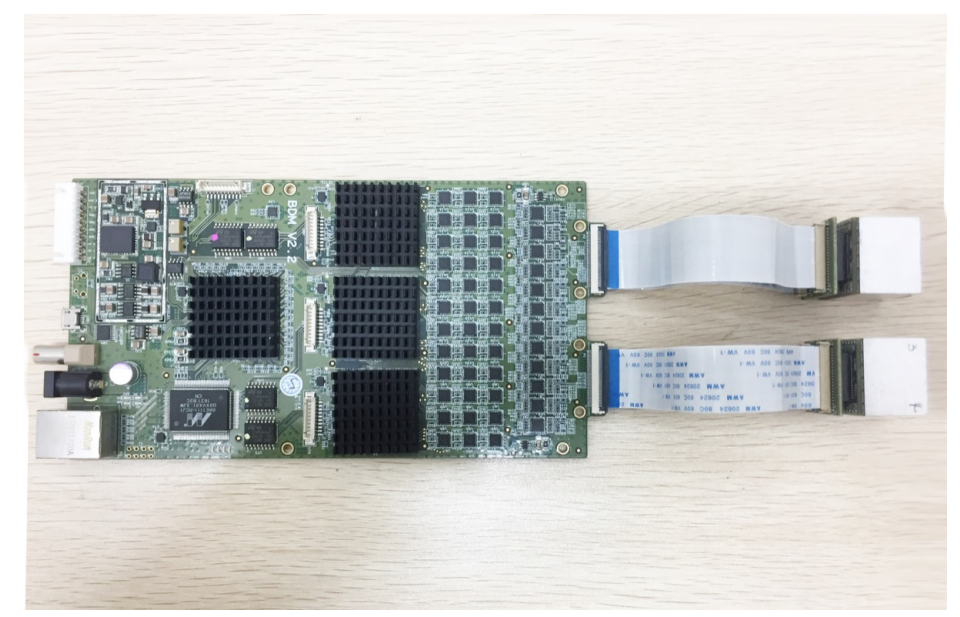

Figure 2. The P\&I sensor system. Two P\&I modules are connected to a MVT readout board. 


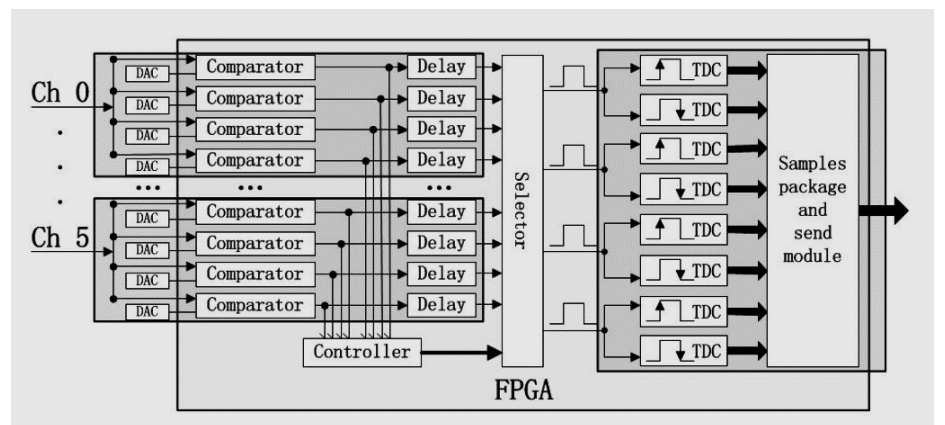

Figure 3. The P\&I sensor system. Schematics of the digital readout electronics based on the MVT concept.

\subsection{The Prototype of PEI PET for Proton Therapy Monitoring}

The P\&I sensor modules described in the previous section were used to design a prototype PET monitoring system for proton therapy. Different views of the design are shown in Figure 4. The prototype consists of two flat panel PET heads composed of a $8 \times 5$ P\&I modules. The total area of each module is approximately $20 \times 12.5 \mathrm{~cm}^{2}$. The total number of LYSO/SiPM channels is 2880 . The distance between the modules is adjustable and is set to $20 \mathrm{~cm}$ in this study. A central support is used to align the target volume with the detector system and place it at the center of the field of view (FOV). As the system is composed of 80 P\&I heads, 40 MVT electronic boards are needed for the readout. A housing support for the boards is placed at both sides of the prototype. A fan cooling system is used to keep the temperature of the prototype stable. Temperature close to the chip is constantly measured, but we did not include a temperature feedback control for the system settings. The whole structure is mounted on a proton therapy patient bed, which can be fixed to the movable stage of the proton therapy room.
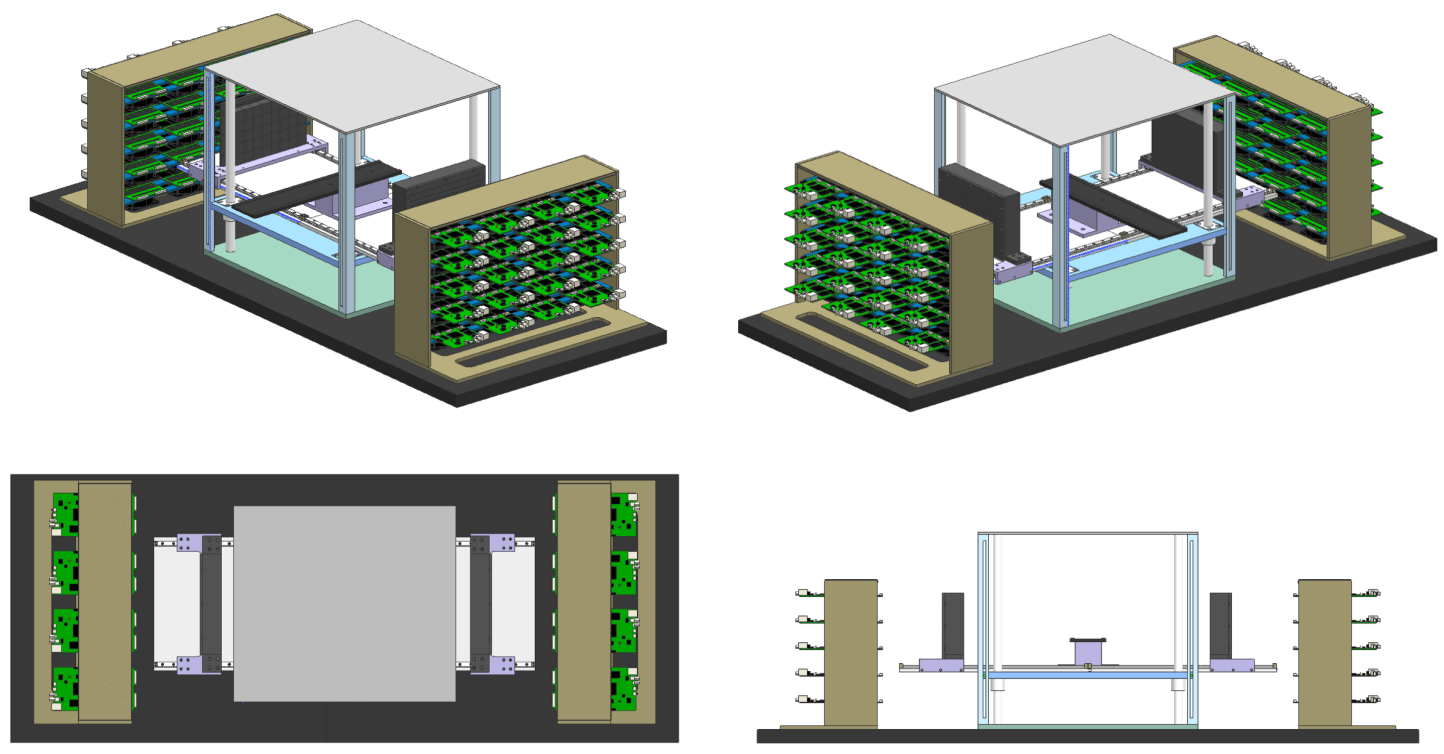

Figure 4. Different views of the design of the prototype of P\&I PET monitoring system for proton beam therapy. The detector modules (flat black panels), the electronic board housing (side structures) and the central positioning support for the alignment of phantoms and detectors are placed on a movable base adaptable to the proton therapy room and fixable to the proton therapy patient's bed.

The prototype was installed in the proton beam treatment room, aligned with the proton beam delivery system, at the proton therapy center of the Chang Gung Memorial Hospital of Taipei. 
A cyclotron generates static $230 \mathrm{MeV}$ proton beams for clinical radiotherapy. An energy degrader and a series of beam instrumentations restrict the beam size and the energy range. After passing through a magnet beam transport system, the beam is delivered with submillimetric precision in the proton therapy room with a rotating gantry base with a diameter of $10.6 \mathrm{~m}$. The installation of the prototype in the treatment room is shown in Figure 5. The prototype was placed on the same robotic coach system used for patients (Figure 5a) and resulted in an alignment with an accuracy of $0.01 \mathrm{~mm}$ after the gantry rotation (Figure $5 b$ ).
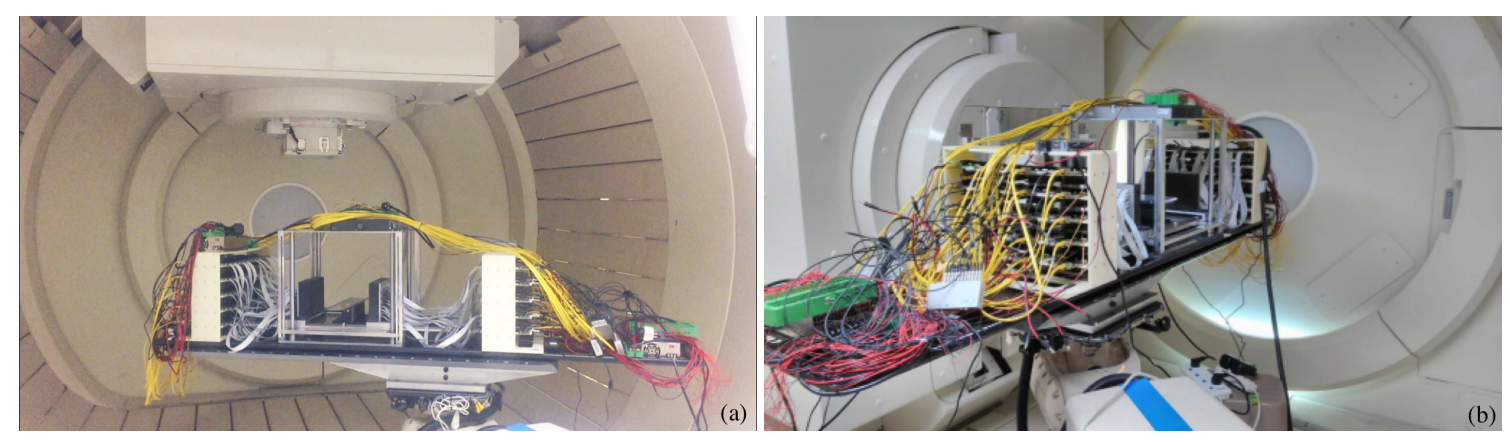

Figure 5. Installation of the prototype of Plug and Imaging sensor PET inside the proton therapy treatment room at the proton therapy center of the Chang Gung Memorial Hospital. The prototype is shown: during the table rotation (a); and in the final position, aligned with the proton beam (b).

\subsection{Phantom Irradiation}

The experiment followed the ethical guidelines and was approved by the Chang Gung Memorial Hospital of Taipei. We prepared a water-filled phantom and a PMMA phantom with density $1.18 \mathrm{~g} / \mathrm{cm}^{3}$ and relative composition of $8.06 \%$ hydrogen, 59.98\% carbon and 31.96\% Oxygen (weight percentages). The phantoms had a transverse section of $5 \times 5 \mathrm{~cm}^{2}$ and a length of $20 \mathrm{~cm}$ along the beam direction. They were irradiated with a $150 \mathrm{MeV}$ pencil beam aligned with its center. The beam had a Gaussian transversal profile with full width half maximum of approximately $2 \mathrm{~mm}$ and $0.3 \%$ energy spread.

The irradiation of the water phantom was performed with high proton statistics of approximately $10^{11}$ protons corresponding to a total dose of approximately $8 \times 10^{3} \mathrm{cGy}$ delivered within a time of approximately $180 \mathrm{~s}$. The irradiation of the PMMA phantom was performed with high proton statistics of approximately $10^{10}$ protons corresponding to a total dose of approximately $800 \mathrm{cGy}$ delivered within a time of approximately $15 \mathrm{~s}$.

Data were acquired during the beam-on time and during the following $3600 \mathrm{~s}$ after the beam is turned off (beam-off period). During the beam-on time, we reached a saturated count rate of 5.2 Mcps. When the beam was off, the count rate decreased to approximately 3.2 Mcps.

Before and after each irradiation, a system calibration run was performed with a phantom containing two ${ }^{22} \mathrm{Na}$ point-like sources placed in the middle plane of the system. The first one was placed in the center of the FOV, and the second one at a distance of $5 \mathrm{~cm}$ along the beam direction. A total statistics of $10^{5}$ hits per channel was collected in 10 min acquisition and was used to verify the operational condition of the system.

\subsection{Data Acquisition and Processing}

The four voltage thresholds needed by the MVT digital readout electronics were set to $20 \mathrm{mV}$, $115 \mathrm{mV}, 210 \mathrm{mV}$ and $305 \mathrm{mV}$ during the acquisition. These settings were tuned to guarantee a time resolution and an energy resolution of approximately $20 \%$ and 500 ps [56]. If a signal in a channel crosses the four thresholds, the eight crossing times measured by the TDC are stored in a raw data file, which contains all acquired hits without any selection. The data processing was performed offline after acquisition. 
The crossing time at the $20 \mathrm{mV}$ threshold was taken as time stamp of the hit and was expressed in ns. The eight samples were fit with the function:

$$
V(t)=a \exp (-b t)(1-\exp (-c t))
$$

The integral of the fitted function within a time window of $200 \mathrm{~ns}$ was taken as the energy of the hit and was expressed in arbitrary units (AU).

The data of calibration runs obtained with the ${ }^{22} \mathrm{Na}$ sources were used to identify for each channel the position and the width of the photoelectric peak corresponding to the $511 \mathrm{keV}$ gamma ray. The energy spectrum of each channel was analyzed. The mean value and the width of the photoelectric peak were estimated with a gaussian fit and the values were stored in a look up table.

The calibration of the channels was used to further process the collected data after phantom irradiation. Singles were formed imposing to the reconstructed hits a symmetric energy window with a total width of $2 \sigma$ around the tabulated value of the photoelectric peak. The position of the singles was taken as the position of the center of the LYSO crystal of the corresponding channel. Finally, coincidences were sorted out of the singles imposing a coincidence time window of $10 \mathrm{~ns}$ for the beam off data and $5 \mathrm{~ns}$ for the beam-on acquisition. The shorter coincidence time allows reducing the random coincidences generated by background photons in the beam on operation. The use of coincidence window as low as $3 \mathrm{~ns}$ is reported in the literature for this application [31]. The lines of response (LOR) were formed using the single position information.

The LORs formed a sinogram, which was subsequently analyzed using an ordered subset expectation-maximization (OSEM) method. The sensitivity correction is approximated calculating the system response matrix with a Monte Carlo solid angle-based technique. Scatter and attenuation corrections are not applied [65]. The reconstructed FOV had a volume of $20 \times 12 \times 20 \mathrm{~cm}^{3}$ segmented into $1 \mathrm{~mm}^{3}$ voxels.

\subsection{Data Analysis}

As stated in Section 1, the key issue in the application of PET as a monitoring system during proton therapy treatment is the reliable reconstruction of the space distribution of the positron emitters produced in the target volume. To address this problem, the data analysis focused on three different aspects of the P\&I sensor for PET proton beam monitoring systems, namely the technical specifications of the prototype, the performance of the prototype after beam irradiation and the response of the prototype in the more challenging scenario of beam-on operation.

At first, we verified that the design of a system composed of such a large number of independent LYSO/SiPM sensors had a uniform response and that its performances were controllable and reproducible in the experimental conditions of the proton therapy room. To this aim, the position and width of the energy spectra of each channel collected in the calibration runs at a time distance of $10 \mathrm{~h}$ were compared.

Secondly, we analyzed the whole set of PMMA data, including the statistics collected during proton irradiation. We extracted the 2D lateral profile and the 1D profile along the beam direction from the reconstructed image.

The typical longitudinal profile of the reconstructed distribution of the positron-emitter nuclei generated by proton irradiation in the target volume is characterized by a rising and falling edge. We fitted the two edges separately with the function:

$$
g(z)=a_{0}+\frac{a_{1}-a_{0}}{1+\exp \left[a_{2}\left(z-a_{3}\right)\right]}
$$

where $a_{0}$ is the background, $a_{1}$ is the maximum value, $a_{2}$ is the fall-off slope and $a_{3}$ is the inflection point. The estimator for the starting point and the ending point of the profile were the values of $a_{3}$ in 
the two fits. The length of the activity profile was calculated as the difference between the fitted $a_{3}$. The width of the falling edge of the profile was estimated as the inverse of the fall-off slope $a_{2}$.

We investigated the possibility of a precise measurement of the exponential decay of the positron emitters produced in the target volume after proton irradiation, as this is also another key-indicator of the reliability of the P\&I sensor for proton therapy monitoring systems. For this purpose, the coincidence rate integrated over the whole FOV was used. A random coincidence subtraction process, based on the delayed window technique, was applied [66]. The average value of $323.5 \mathrm{~Hz}$ random coincidence rate was subtracted statistically from the measured rate, using the Poisson counting statistics approximation. The coincidence rate measured in the beam-off state corresponds to the decay of the positron emitters produced in the target volume during proton irradiation. We restricted the analysis to the most abundant elements produced in the experimental conditions of this paper, namely ${ }^{15} \mathrm{O},{ }^{11} \mathrm{C}$ and ${ }^{10} \mathrm{C}$. The respective production channels and thresholds are reported in Table 1 following [67].

Table 1. Most relevant positron emitters produced in the target volume and production channels used in this study.

\begin{tabular}{ccc}
\hline Isotope & Channel & Threshold [MeV] \\
\hline${ }^{15} \mathrm{O}$ & ${ }^{16} \mathrm{O}(\mathrm{p}, \mathrm{pn}){ }^{15} \mathrm{O}$ & 16.79 \\
${ }^{11} \mathrm{C}$ & ${ }^{12} \mathrm{C}(\mathrm{p}, \mathrm{pn}){ }^{11} \mathrm{C}$ & 20.61 \\
& ${ }^{16} \mathrm{O}(\mathrm{p}, 3 \mathrm{p} 3 \mathrm{n}){ }^{11} \mathrm{C}$ & 59.64 \\
${ }^{10} \mathrm{C}$ & ${ }^{12} \mathrm{C}(\mathrm{p}, \mathrm{p} 2 \mathrm{n}){ }^{10} \mathrm{C}$ & 35 \\
& $\left.{ }^{16} \mathrm{O}(\mathrm{p}, 3 \mathrm{p} 4 \mathrm{n})\right)^{10} \mathrm{C}$ & 72 \\
\hline
\end{tabular}

We fitted the time-dependent coincidence rate with a function consisting of the sum of exponentials:

$$
f(t)=A_{\left({ }^{15} \mathrm{O}\right)} \exp \left[-t / \tau_{\left({ }^{15} \mathrm{O}\right)}\right]+A_{\left({ }^{11} \mathrm{C}\right)} \exp \left[-t / \tau_{\left({ }^{11} \mathrm{C}\right)}\right]+A_{\left({ }^{10} \mathrm{C}\right)} \exp \left[-t / \tau_{\left({ }^{10} \mathrm{C}\right)}\right]
$$

The fit was calculated in the range between $t_{1}=1 \mathrm{~s}$ and $t_{2}=2000 \mathrm{~s}$ after the end of the beam irradiation. The amplitude $A_{i}$ and the half-life $\tau_{i} / \ln 2$ of each element were the free parameters of the fit.

The relative abundance $C_{i}$ of each element at the end of the irradiation was calculated from the fit results using the formula:

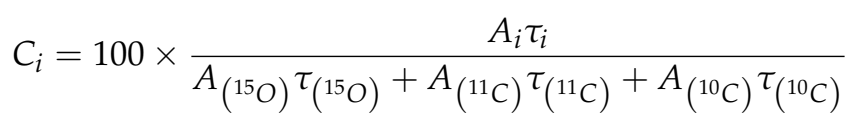

The statistical uncertainty of $C_{i}$ was calculated from the errors of the parameters $A_{i}$ and $\tau_{i}$ estimated in the fit using the standard error propagation technique.

Finally, we investigated the performances of the prototype in the more challenging scenario of the beam-on operation by analyzing the beam-on water phantom data. We studied the specific features of the energy spectra measured in the detector and we extracted the 2D lateral profile and the 1D profile along the beam direction from the reconstructed image. The analysis of the measured profile of the produced positron emitting nuclides is performed using the same technique described above in Equation (2).

To verify whether the measured profiles and nuclide abundance follow the physical expectations, we simulated the interaction of the proton beam with the targets. We used the simulation framework GEANT4, following the suggestions in [29]. The physics list included quark gluon string precompound (QGSP) hadronic model for nucleons, binary ion cascade (BIC) inelastic model for ions and high precision model built upon the TALYS-based evaluated nuclear data library (TENDL) for isotope 
production. For electromagnetic interactions, we used the available electromagnetic option for medical applications.

A pencil proton beam with energy $150 \mathrm{MeV}$, energy spread $0.3 \mathrm{MeV}$ and transversal width $2 \mathrm{~mm}$ was simulated, which is an approximation of the expected features of the proton beam used in the experiment.

As for the analysis of the space distribution of positron emitters in the target volume, we estimated the length and the falling edge width $\sigma_{\text {phys }}$ of the profile of the secondary positron emitters produced in the target volume irradiation using the same fitting technique in Equation (2). The length of the profile was compared directly with the measured value. We interpreted the measured width $\sigma_{\text {meas }}$ as the combination of the physical width $\sigma_{\text {phys }}$ and of the experimental factors $\sigma_{\text {exp }}$, including detector and scanner geometry, reconstruction, random coincidences and noise. The two contributions are independent and they sum up in quadrature:

$$
\sigma_{\text {meas }}^{2}=\sigma_{\text {phys }}^{2}+\sigma_{\text {exp }}^{2}
$$

The experimental contribution to the width was thus estimated as:

$$
\sigma_{\text {exp }}=\sqrt{\sigma_{\text {meas }}^{2}-\sigma_{\text {phys }}^{2}}
$$

As for the nuclide analysis, we calculated the relative abundance of the produced ${ }^{15} \mathrm{O},{ }^{11} \mathrm{C}$ and ${ }^{10} \mathrm{C}$ positron emitters directly storing the physics process information provided by the stepping action during the simulation. The physical expectations obtained in the simulation were compared with the experimental results.

\section{Results}

\subsection{The Performance of the PEI Sensor System}

The eight samples generated by the MVT digitizer for a hit pulse in a LYSO/SiPM channel during a ${ }^{22} \mathrm{Na}$ calibration run are shown in Figure 6a. The continuous red line in the figure is the estimation of the original signal through the fitting function in Equation (1). The electronic noise introduces a baseline pedestal in the integral spectrum, which is estimated in dedicated noise runs with lowered thresholds. The pedestal-subtracted distribution of a sub-sample of 30,000 events registered in a SiPM/LYSO channel in response to a ${ }^{22} \mathrm{Na}$ source during the calibration run is shown in Figure $6 \mathrm{~b}$. The spectrum exhibits the typical features of the detection of the $511 \mathrm{keV}$ gamma-ray: a photoelectric peak at approximately $25000 \mathrm{AU}$ and a Compton continuum at lower energies. Since a signal is stored only if all four voltage thresholds are crossed, the energy spectrum exhibits a lower threshold at approximately $14,000 \mathrm{AU}$.

The position of the $511 \mathrm{keV}$ photoelectric peak $\left(\mathrm{ph}_{\mathrm{pos}}\right)$ measured during a $10 \mathrm{~min}^{22} \mathrm{Na}$ calibration run in the 2880 detector channels is shown in Figure 7a. We registered a temperature fluctuation of approximately $1{ }^{\circ} \mathrm{C}$ (FWHM) during the acquisition. The error bars represent the width of the photoelectric peak. We observe that the position of the photoelectric peak ranges between 25,000 and 30,000 in all channels. The FWHM of the photoelectric peak is ranging between $5500 \mathrm{AU}$ and 10,000 AU. The distribution of the energy resolution in the 2880 channels composing the proton therapy monitoring PET prototype is reported in Figure 7b. The SiPM/LYSO sensor channels with MVT readout show

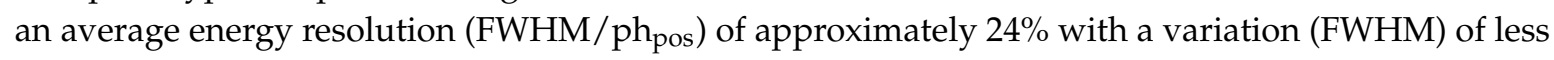
than $5 \%$ across the system.

A 2D histogram of the energy resolution obtained in the two modules of the prototype is shown in Figure 8. We do not observe any sizeable position dependence.

To verify the possibility to control the device in the experimental conditions of the proton therapy room, we plot the position of the $511 \mathrm{keV}$ photoelectric peak taken at a time distance of ten hours in Figure 9. Each data point in the plot corresponds to one of the 2880 independent channels. We registered 
a temperature increase of approximately $10{ }^{\circ} \mathrm{C}$ close to the sensors between the two measurements. The position of the peak exhibits a reduction of approximately $15 \%$. The gain and the photon detection efficiency of the SiPM are in fact decreasing with increasing temperature. We measure temperature variations between different runs up to $25^{\circ} \mathrm{C}$, depending on the working time period of the system.
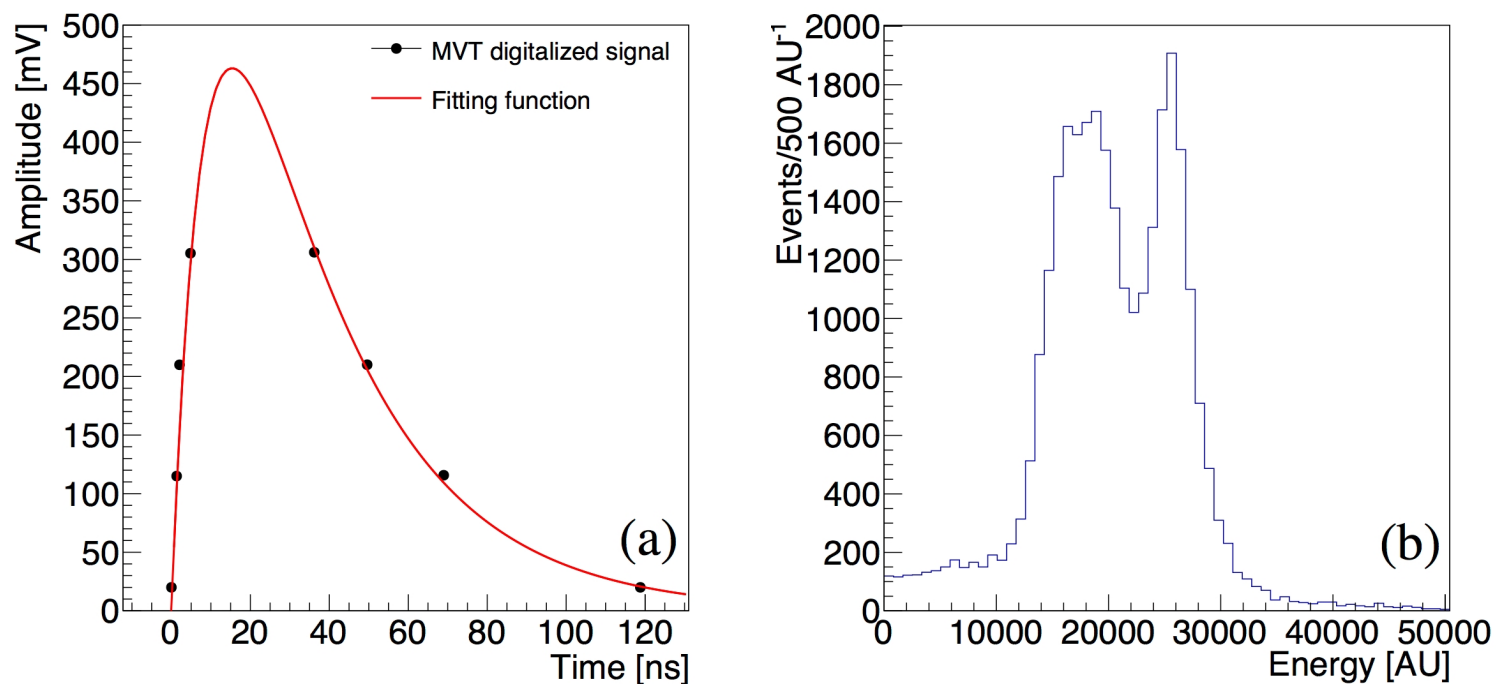

Figure 6. Response of a LYSO/SIPM channel after the detection of a $511 \mathrm{keV}$ photon from a ${ }^{22} \mathrm{Na}$ source. In (a) the MVT digitized signal (black points) and the fitted function reconstructing the original signal (red line) are shown. The fitted function is integrated within a time window of $200 \mathrm{~ns}$ and the distribution of its integral for a small sub-sample of 30,000 events is shown in (b): the photoelectric peak and the Compton continuum are visible in the obtained spectrum.
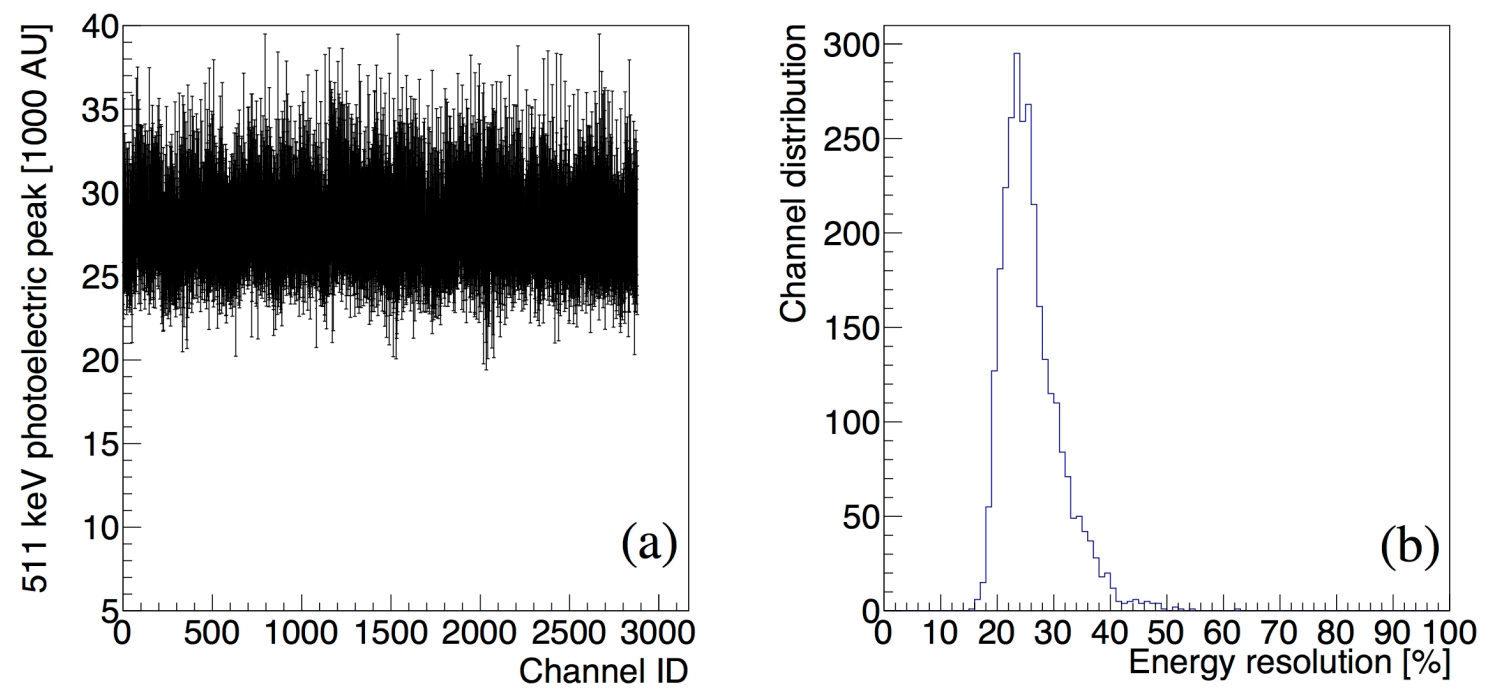

Figure 7. System calibration: position $\left(\mathrm{ph}_{\mathrm{pos}}\right)(\mathbf{a})$ and energy resolution $(\mathrm{FWHM} / \mathrm{ph}$ pos $)(\mathbf{b})$ of the $511 \mathrm{keV}$ photoelectric peak measured during a ${ }^{22} \mathrm{Na}$ calibration run in the 2880 channels. 

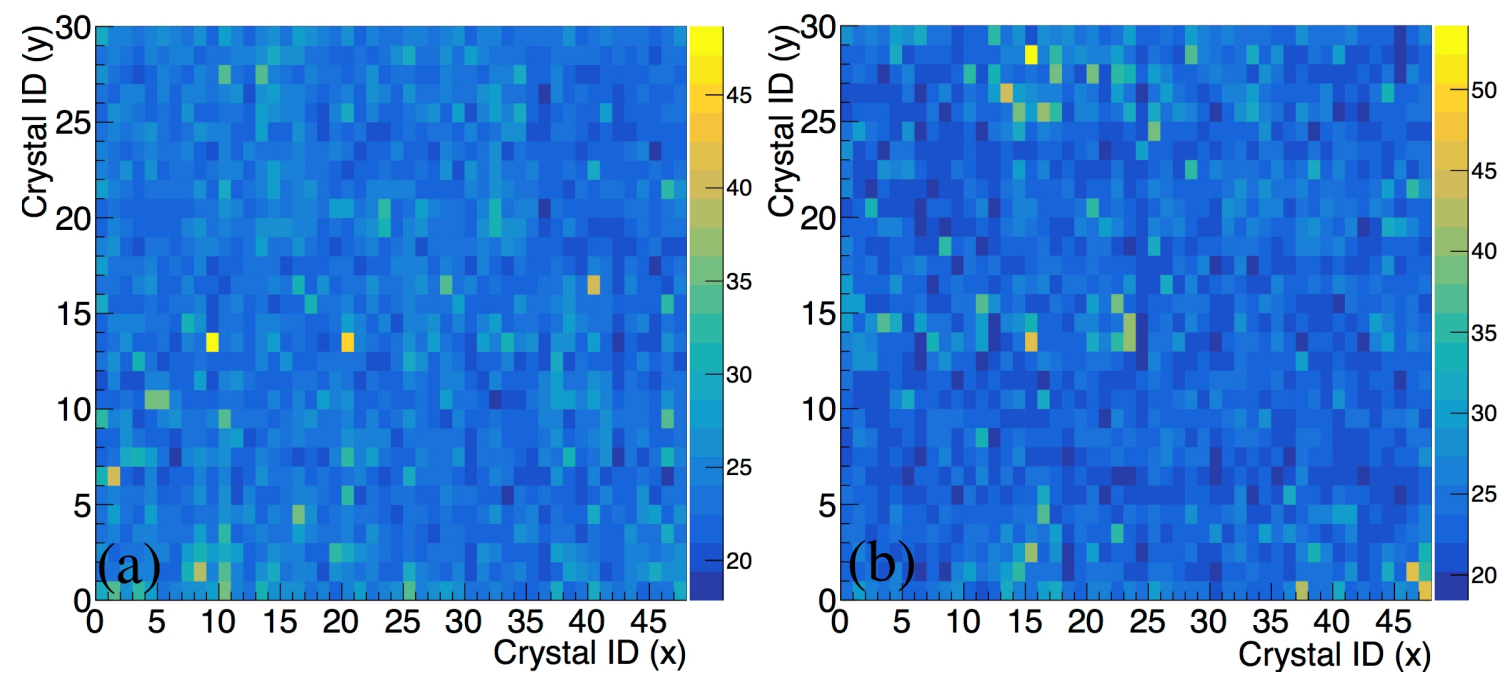

Figure 8. $2 \mathrm{D}$ histogram of the energy resolution $\left(\mathrm{FWHM} / \mathrm{ph} \mathrm{pos}_{\mathrm{s}}\right)$ obtained in the prototype PET monitoring system for the left (a) and right (b) module with respect to the beam direction.

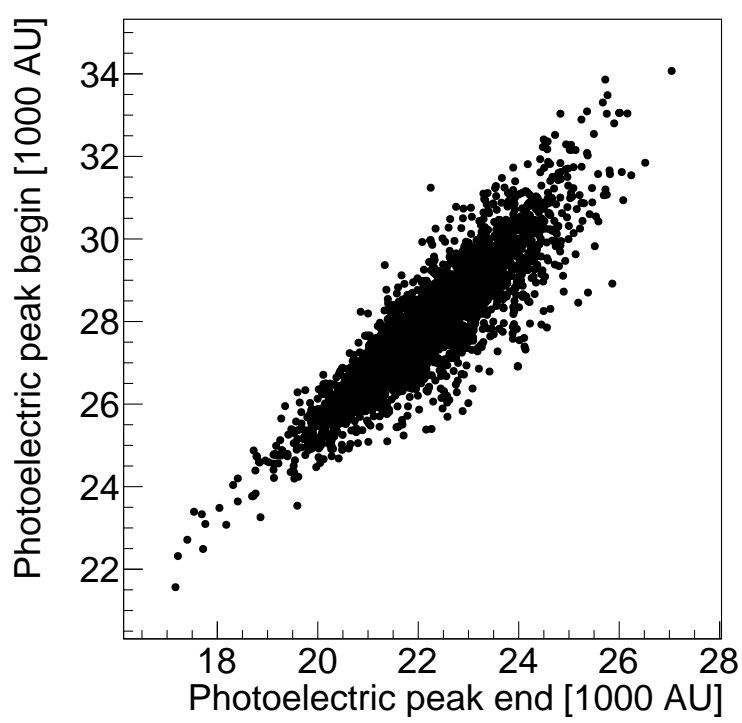

Figure 9. Position of the $511 \mathrm{keV}$ photoelectric peak taken at a time distance of ten hours.

\subsection{Lateral Profile of the Positron-Emitting Nuclei}

The reconstructed measured lateral profile of the positron emitters produced in the PMMA phantom after a $150 \mathrm{MeV}$ proton irradiation is shown in Figure 10a. The width of the activity distribution in the $y$-direction perpendicular to the beam axis reflects the beam spot size, the scattering of the secondary particles produced in the target volume and the intrinsic experimental resolution of the detection system. The gray area corresponds to the reconstructed field of view. The one-dimensional profile of the induced positron activity along the beam $z$-axis at the central position $y=10 \mathrm{~cm}$ is shown in Figure 10b.

The start-point and end-point of the profile are estimated with the fitting function in Equation (2) as, respectively, $a_{3, s}=(0.77 \pm 0.32) \mathrm{cm}$ and $a_{3, e}=(14.10 \pm 0.23) \mathrm{cm}$. The estimated length of the activity profile is $a_{3, e}-a_{3, s}=(13.33 \pm 0.55) \mathrm{cm}$ and is in good agreement with the value $(13.57 \pm 0.02) \mathrm{cm}$ expected from the simulation. 

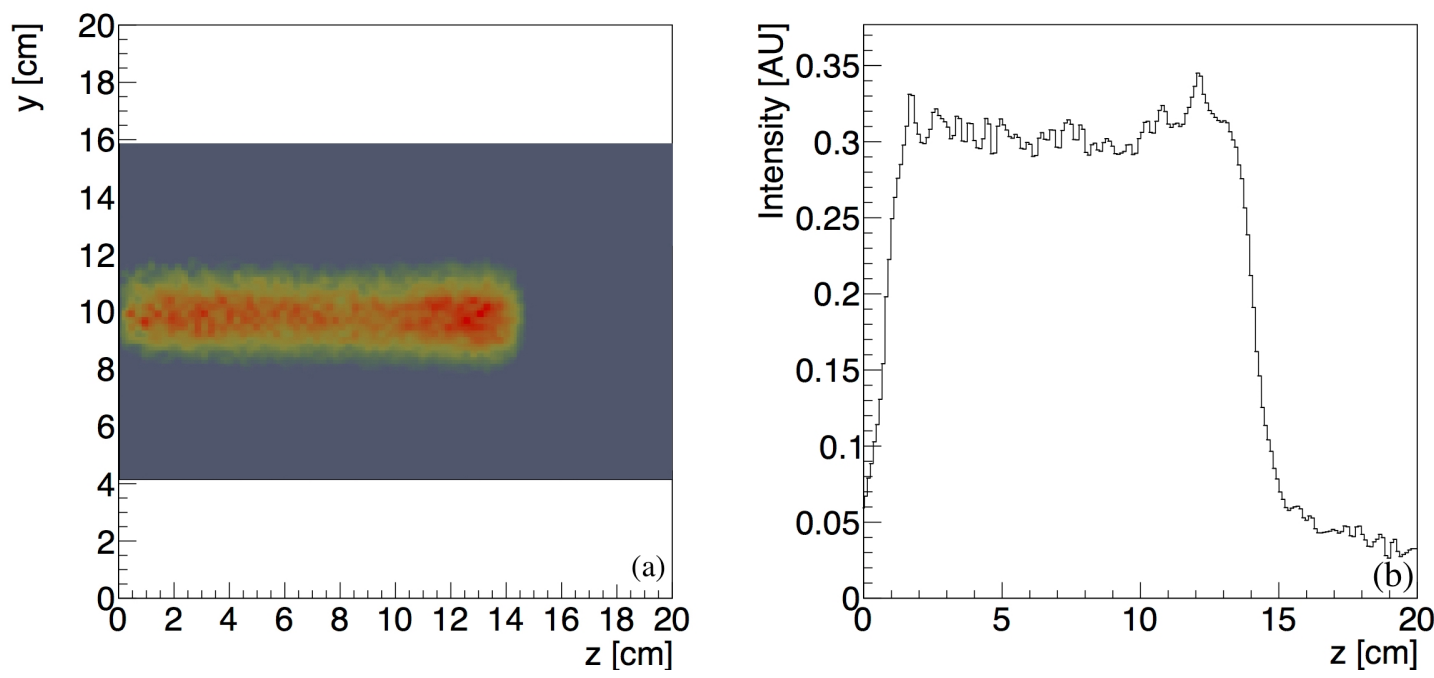

Figure 10. Reconstructed lateral profile at $y=10 \mathrm{~cm}(\mathbf{a})$; and one-dimensional longitudinal profile (b) of the positron emitters produced by a $150 \mathrm{MeV}$ proton pencil beam with approximately $2 \mathrm{~mm}$ spot-size and $0.3 \mathrm{MeV}$ energy spread within a PMMA target volume.

The starting-edge of the profile has a width of approximately $1 \mathrm{~cm}$ due to the acceptance limitations at the borders of the FOV. The Bragg peak appears smeared with a width estimated as $\sigma_{\text {meas }}=(0.33 \pm 0.01) \mathrm{cm}$. The width expected from the simulation is $\sigma_{\text {phys }}=(0.16 \pm 0.11) \mathrm{cm}$. By comparing the measured and the physical width, following Equation (6), we estimated an experimental contribution of $\sigma_{\text {exp }}=(2.88 \pm 0.03) \mathrm{mm}$.

\subsection{Time Distribution of the Coincidence Rate}

The decay half life and the simulated abundance of the ${ }^{15} \mathrm{O},{ }^{11} \mathrm{C}$ and ${ }^{10} \mathrm{C}$ positron emitters as estimated in the simulation are reported in the second and fourth columns of Table 2, respectively.

Table 2. Relative abundance and half life of the isotopes produced after proton irradiation in the Monte Carlo simulation and experimental results after fitting the measured coincidence rate.

\begin{tabular}{ccccc}
\hline Element & $\begin{array}{c}\text { Half life } \\
\text { Literature } \\
{[\mathbf{s}]}\end{array}$ & $\begin{array}{c}\text { Half Life } \\
\text { Measurement } \\
{[\mathbf{s}]}\end{array}$ & $\begin{array}{c}\text { Abundance } \\
\text { Simulation } \\
\mathbf{( \% )}\end{array}$ & $\begin{array}{c}\text { Abundance } \\
\text { Measurement } \\
(\%)\end{array}$ \\
\hline${ }^{15} \mathrm{O}$ & 122 & $121.8 \pm 0.6$ & $27 \pm 2$ & $25 \pm 3$ \\
${ }^{11} \mathrm{C}$ & 1220 & $1218 \pm 6$ & $70 \pm 3$ & $73 \pm 2$ \\
${ }^{10} \mathrm{C}$ & 19.3 & $19.13 \pm 0.09$ & $3.0 \pm 0.2$ & $2.6 \pm 0.2$ \\
\hline
\end{tabular}

The measured coincidence rate is shown in Figure 11. The data points were fitted with the function in Equation (3). The total fitting function and each component are displayed in the plot. The fit results are reported in Table 2. The value of the half-life of the three positron emitters was estimated with a relative accuracy of approximately $0.5 \%$ and was found in good agreement with the literature values used in the simulation.

The relative abundance of the produced positron emitters was calculated from the fit results using Equation (4) and it is shown in the last column of Table 2. It was estimated with a relative accuracy of approximately $5 \%$ and the results were found in good agreement with the expectation from simulation. 


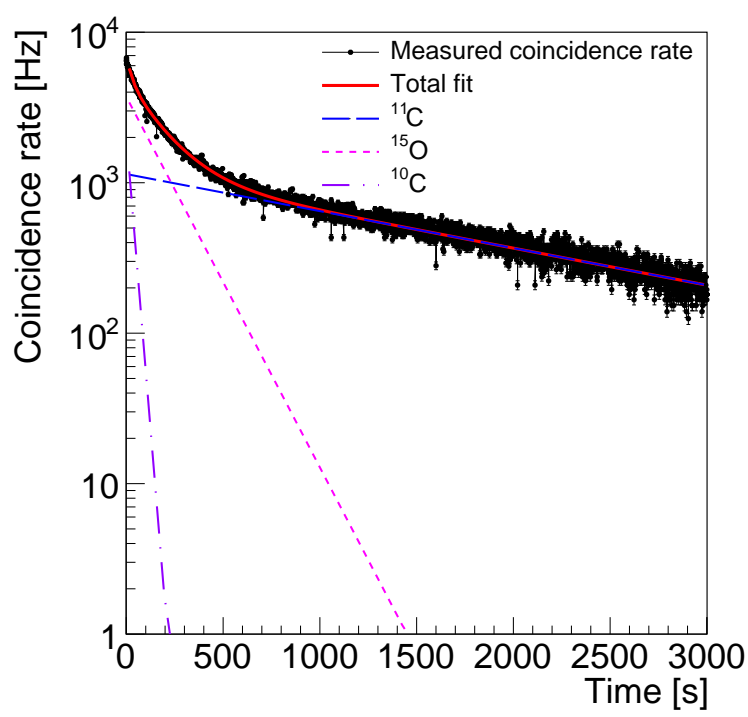

Figure 11. Time dependence of the measured coincidence rate. The exponential decay fitting function and the separate components corresponding to the activated elements are shown.

\subsection{Prototype Performance in Beam-On Operation}

The signal measured in the prototype after irradiation is generated by the decay of the positron emitting nuclides produced in the target sample. The condition in beam-on operation changes significantly. In fact, a physical background generated by prompt photons arises from nuclear interactions and beam-induced radiation in the experimental area.

The effect of the background is shown in Figure 12 for the case of the water phantom irradiated by a $150 \mathrm{MeV}$ proton beam with irradiation conditions specified in Section 2.3. The blue histogram represents the distribution of the energy measured in one channel of the prototype during beam irradiation without the coincidence time window. The peak corresponding to the photoelectric detection of $511 \mathrm{keV}$ photons is at approximately 20,000 A.U. The Compton component of the spectrum is represented by the peak at approximately 15,000 A.U, due to the sculpting effect of the threshold. In addition, further highly energetic peaks, such as at approximately $30,000 \mathrm{AU}$, are present in the spectrum, corresponding to proton-beam induced background. The response of the system to high energies is not linear and it is not possible to estimate with precision the energy correspondent to the highly energetic peaks. The red histogram represents the energy distribution of the events after time coincidence selection. We observe that most of the beam-induced background is removed when only signals are taken from one of the crystals, which are in $5 \mathrm{~ns}$ time coincidence with any other crystal in the opposite panel. The photoelectric peak and the Compton structure are well visible after event selection.

The reconstructed measured lateral profile of the positron emitters produced in the water phantom during a $150 \mathrm{MeV}$ proton irradiation is shown in Figure 13a. The width of the activity distribution in the $y$-direction perpendicular to the beam axis reflects the beam spot size, the scattering of the secondary particles produced in the target volume and the intrinsic experimental resolution of the detection system. The gray area corresponds to the reconstructed field of view. The one-dimensional profile of the induced positron activity along the beam $z$-axis at the central position $y=10 \mathrm{~cm}$ is shown in Figure 13b.

The start-point and end-point of the profile are estimated with the fitting function in Equation (2) as, respectively, $a_{3, s}=(0.80 \pm 0.21) \mathrm{cm}$ and $a_{3, e}=(14.22 \pm 0.30) \mathrm{cm}$. The estimated length of the activity profile is $a_{3, e}-a_{3, s}=(13.42 \pm 0.51) \mathrm{cm}$ and is in good agreement with the value $(13.30 \pm 0.02) \mathrm{cm}$ expected from the simulation. 


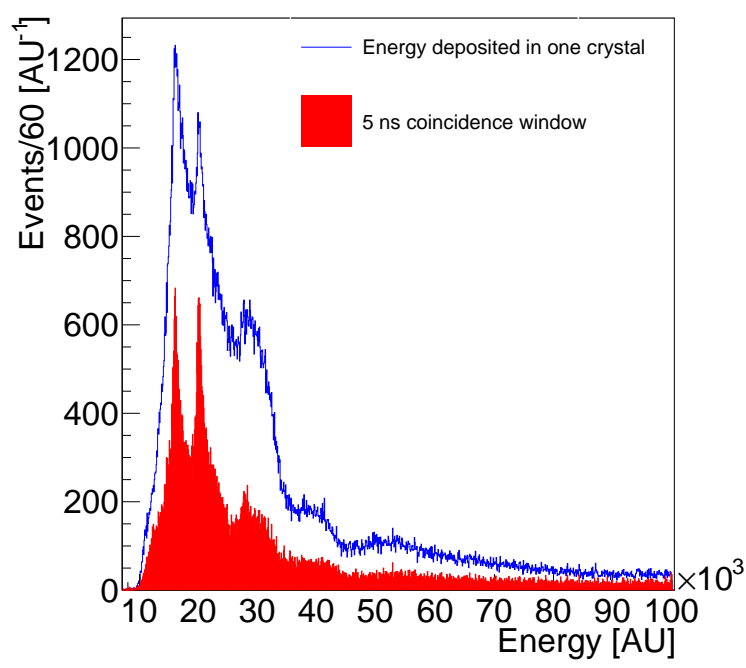

Figure 12. Histogram of the energy deposited in one of the crystals of the PET monitoring system during beam-on operation before (blue line) and after (filled red) 5 ns time coincidence.
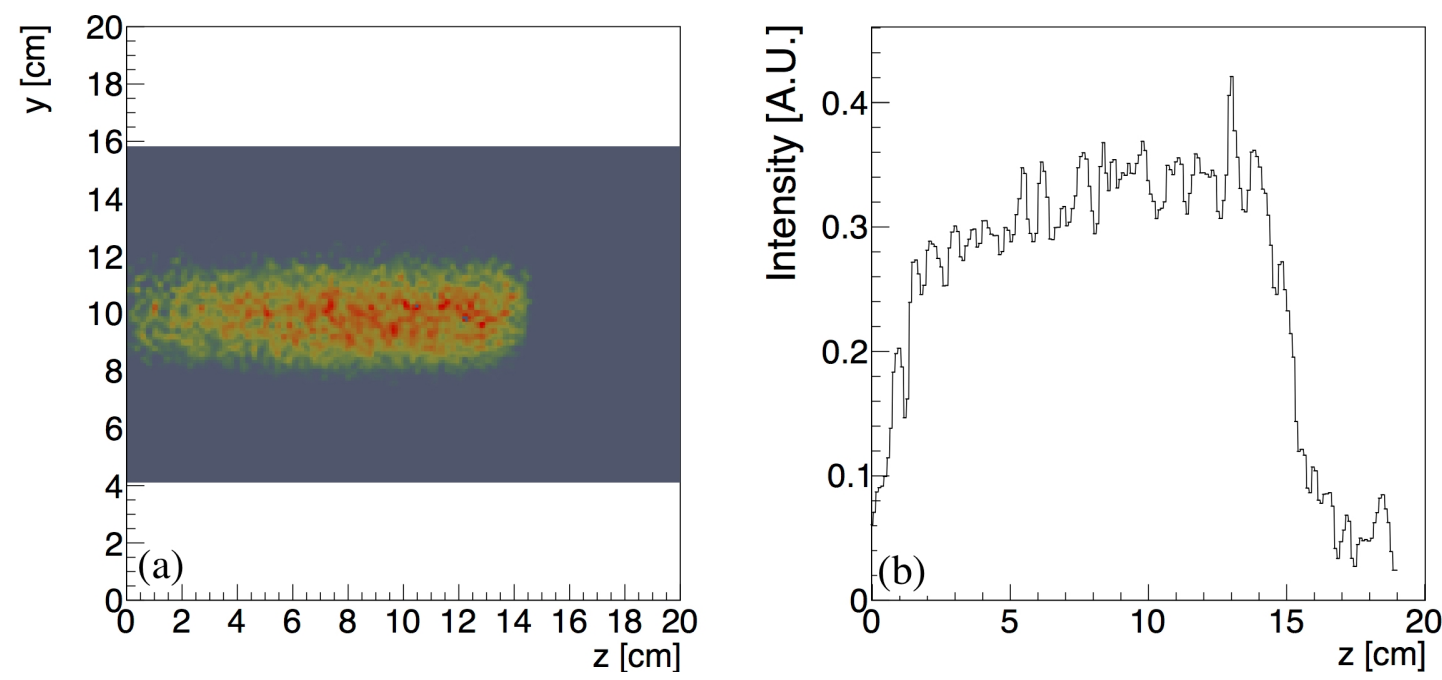

Figure 13. Reconstructed lateral profile at at $y=10 \mathrm{~cm}$ (a); and one-dimensional longitudinal profile (b) of the positron emitters produced by a $150 \mathrm{MeV}$ proton pencil beam with approximately $2 \mathrm{~mm}$ spot-size and $0.3 \mathrm{MeV}$ energy spread within a water target volume during beam-on operation.

\section{Discussion}

We tested a prototype of PET monitoring system for proton therapy obtained with the P\&I sensor based on the LYSO/SiPM optical sensors. The size of the prototype compares well with other systems developed in previous studies. Panel PET prototypes with size ranging between $6 \times 6 \mathrm{~cm}^{2}$ and $10 \times 25 \mathrm{~cm}^{2}$ are reported in the literature [30,31,36-38]. In line with the most recent advances of this technique, we performed the experiment in-beam, during both beam-on and beam-off time.

The key improvement obtained in this paper is the unique digital readout scheme applied to the LYSO/SiPM sensor system. The MVT technique proposed here offers a possible alternative both to the constant $1 \mathrm{GHz}$ sampling [68-74] and to the single threshold approach [38]. All PET monitoring systems based on scintillator/SiPM sensing technology suffer from intrinsic limitations in count rate determined by the proper decay time of the chosen scintillator. However, the digitization stage of the readout electronics usually imposes the most stringent limitation to the maximal allowed count rate. The novel sensor readout configuration specifically designed for SiPM-based systems allowed 
reaching a maximal count rate ranging between $0.25 \mathrm{Mcps}$ [36] and 3.5 Mcps [38], due to limitations in readout architecture and data transfer solutions. The count rate limitation of a PET monitoring system based on one of the most advanced SiPM (PHILIPS) is reported to be 1.3 Mcps [75]. The saturation count rate obtained in this study during the beam-on time is $5.2 \mathrm{Mcps}$ and overwhelms the above limits significantly. The imaging of proton induced activity in the beam-on condition is possible with the P\&I sensor system.

The high count rate is obtained reducing the sampling frequency. Clearly, having only eight samples available, the digital signal processing capabilities and analysis techniques are limited with respect to the $1 \mathrm{GHz}$ sampling frequency technology [68]. This has an impact on the energy resolution of the $511 \mathrm{keV}$ photoelectric peak. We measured an energy resolution of approximately $23 \%$, against the $12-14 \%$ obtained in other PET monitoring systems based on scintillator/SiPM sensing technology with different readout electronics [30,31,36-38]. This result indicates that a better fitting strategy than the one proposed in Equation (1) needs to be investigated to improve the energy resolution of the PET monitoring system.

Besides the optimization of the fitting strategy, we found that a better cooling of the system is needed. Both the detector heads and the MVT boards have sizeable temperature fluctuations. SiPM sensors are based on the impact-ionization process, the probability of which decreases with increasing temperature. Thus, a higher internal electric field is needed in the SiPM structures for higher temperature. When the temperature has a positive change and the bias voltage is kept fixed, the breakdown voltage of the SiPM sensors increases and the gain and the photon detection efficiency of the SiPM sensors decreases. As a result, the position of the $511 \mathrm{keV}$ photoelectric peak has a negative shift when the temperature increases. The changes in the gain during the data acquisition deteriorates the energy resolution of the detector. This is a common feature of all SiPM-based compact systems and can be controlled with periodic calibration runs, temperature correction of the calibration constants, as performed for example in [76] and with a dedicated cooling system as in [36].

Moreover, the four voltage thresholds need to be adjusted according to the changing signal amplitude. Currently, they are fixed according to a characterization performed in the laboratory environment in the production stage, which is optimal for the operation of traditional Positron Emission Tomography systems with controlled temperature. The large energy resolution measured in the prototype suggests that an optimization of the voltage sampling thresholds is needed to adapt the sampling to the real-time amplitude of the LYSO/SiPM signals.

Another effect to be further investigated is the deterioration of the tensile strength of the optical glue, due to both local temperature changes above allowed temperature limits and exceeding shear stress. Consequently, the optical coupling between crystals and SiPMs deteriorates and the energy resolution is subject to degradation. The results suggest a verification of the used optical glue and a comparison with other available glues.

The LYSO/SiPM sensing channels constituting the P\&I system exhibit a non-uniformity of less than $5 \%$ in both position and width of the $511 \mathrm{keV}$ photoelectric peak. This result shows that the SiPM technology is mature for the construction of large scale systems, as already noted in [37], where a 10\% non-uniformity of a LYSO/SiPM-based PET system for proton therapy monitoring is reported.

As for the longitudinal profile of the reconstructed positron emitters activity in the target volume after irradiation, we obtained a spatial resolution of approximately $3 \mathrm{~mm}$ (FWHM), which is consistent with the size of the pixels of the prototype. This accuracy is following the improvement of the performances for PET monitoring systems provided with the SiPM. However, SiPM-based PET monitor systems exhibited better values, ranging between $1.5 \mathrm{~mm}$ and $3 \mathrm{~mm}$ [30,31]. This suggests that the cross-sectional size of the LYSO/SiPM detection system can be decreased to improve the spatial accuracy. Besides these hardware considerations, the measured longitudinal length of the positron emitters distribution in the target volume was consistent with the simulation expectations. However, a proper comparison between the features of the profiles in measurement and in simulation 
requires introducing in the simulation the geometry of the detector, the properties of the electronic chain for the generation of the electric signals and the reconstruction of the simulated digitized hits [67].

Finally, the time stamping obtained with the MVT method applied to the LYSO/SiPM detection system allows reconstructing both the decay and the relative abundance of the positron-emitters ${ }^{15} \mathrm{O}$, ${ }^{11} \mathrm{C}$ and ${ }^{10} \mathrm{C}$ produced in the phantom with the irradiation of $10^{10}$ protons in approximately $15 \mathrm{~s}$ with relative accuracies of $0.5 \%$ and $5 \%$, respectively. Our results are in agreement with previous studies $[7,13,14,19,31]$. While maximal coincidence rates ranging between $100 \mathrm{~Hz}$ and $2 \mathrm{kHz}$ are reported in the nuclide analysis of the decay, we observe a maximal measured coincidence rate of $7 \mathrm{kHz} 1 \mathrm{~s}$ after the beginning of the beam-off time. The larger measured coincidence rate statistics is a direct consequence of the higher count rate capability of the P\&I sensor system presented in this paper with respect to other prototypes. Recent studies have shown that SiPM-based PET monitoring systems can resolve also positron emitters with shorter half life, as ${ }^{8} \mathrm{~B}(0.7 \mathrm{~s})$ [37] and ${ }^{12} \mathrm{~N}(11 \mathrm{~ms})$ [36], in the inter-spill times during proton irradiation. The accuracy of the results obtained in this paper and the larger collected statistics suggests that the immediate future direction of our study is a dedicated experiment for the identification and reconstruction of fast-decaying positron emitters produced in the target volume during proton irradiation.

\section{Conclusions}

In the present study, we measured the performance of a the P\&I sensor technology based on LYSO/SiPM systems with digital MVT readout used in a PET monitoring system for proton therapy both in beam-on and in beam-off operational conditions. The key property of the P\&I concept is the higher maximal allowed count rate with respect to other available sensing technologies. The spatial resolution of the prototype is approximately $3 \mathrm{~mm}$ (FWHM). The count rate capability of the MVT electronics guarantees collecting good statistics for precise estimation of the decay time and relative abundance of the produced positron emitters with accuracies of approximately $0.5 \%$ and $5 \%$, respectively, after irradiation with $10^{10}$ proton within approximately $15 \mathrm{~s}$.

The results show that the P\&I sensor is mature for application to non standard PET systems for the monitoring of the delivered dose during proton therapy treatment. A drawback of the sensors is the relatively large space occupied by the digital readout boards. We are currently developing new SiPM sensors in CMOS technology that will allow integrating most of the MVT electronics on the chip, thus improving the compactness of the system design.

Author Contributions: N.D. and Q.X. conceived and designed the experiments; W.W. designed the phantom and the energy of the experiment; N.D., M.G. and Q.X. realized the PET prototype and performed the experiments; H.H.C., F.H.C., J.H.H., I.T.H. and T.C.Y. managed the proton beam and controlled the proton beam conditions during regular $8 \mathrm{~h}$ shifts; D.X. and B.Z. provided the P\&I heads and checked their performance before the assembling of the prototype; N.D. and M.G. analyzed the data; and N.D., P.G. and E.A. analyzed the beam-on data; N.D. wrote the paper. N.D. and M.G. are both first authors of the paper.

Funding: This work was supported in part by the Natural Science Foundation of China (NSFC) (Grants 61425001, 61210003, and 61604059); in part by the Natural Science Foundation of Hubei Province (Grant 2016CFA005); in part by National Key Scientific Instrument and Equipment Development Program of China (2013YQ030923); in part by National Key Scientific Instrument and Equipment Development Program of Hubei Province (2013BEC050); in part by National Key Research and Development Program of China (2016YFF0101500).

Acknowledgments: We acknowledge the help from the Particle Physics and Irradiation Core Laboratory and Radiation Biology Core Laboratory, Chang Gung Memorial Hospital at Linkou.

Conflicts of Interest: The authors declare no conflict of interest. The founding sponsors had no role in the design of the study; in the collection, analyses, or interpretation of data; in the writing of the manuscript, and in the decision to publish the results. 


\section{Abbreviations}

The following abbreviations are used in this manuscript:

$\begin{array}{ll}\text { PET } & \text { Positron Emission Tomography } \\ \text { P\&I } & \text { Plug\&Imaging } \\ \text { DCR } & \text { Dark Count Rate } \\ \text { SiPM } & \text { Silicon Photomultiplier } \\ \text { CMOS } & \text { Complementary Metal Oxide Semiconductor } \\ \text { LYSO } & \text { Lutetium Yttrium Orthosilicate } \\ \text { MVT } & \text { Multi Voltage Threshold } \\ \text { TDC } & \text { Time to Digital Converter } \\ \text { LVDS } & \text { Low Voltage Differential Signaling } \\ \text { FPGA } & \text { Field Programmable Gate Array } \\ \text { FOV } & \text { Field Of View } \\ \text { PMMA } & \text { Polymethylmethacrylat } \\ \text { AU } & \text { Arbitrary Units } \\ \text { OSEM } & \text { Ordered subset expectation maximization }\end{array}$

\section{References}

1. Colletti, P.M. 2017 SNMM: Highlights Lecture: General Nuclear Medicine. J. Nucl. Med. 2017, 58, 11N-15N. [PubMed]

2. D'Ascenzo, N.; Xie, Q. Silicon Photomultiplier: Physics, Technological Challenges and Application to Modern Nuclear Medicine, in Photon Counting; Britun, N., Nikiforov, A., Eds.; INTECH: Rijeka, Croatia, 2018; ISBN 978-953-51-5775-5.

3. Pawelke, J.; Haberer, T.; Hasch, B.G.; Hinz, R.; Kramer, M.; Lauckner, E.; Sobiella, M. In-beam PET imaging for the control of heavy-ion tumour therapy. IEEE Trans. Nucl. Sci. 1997, 44, 1492-1498. [CrossRef]

4. Enghardt, W.; Debus, J.; Haberer, T.; Hasch, B.G.; Hinz, R.; Jäkel, M.; Krämer, O.; Lauckner, K.; Pawelke, J.; Pönisch, F. Positron emission tomography for quality assurance of cancer therapy with light ion beams. Nucl. Phys. A 1999, 654, 1047-1050. [CrossRef]

5. Enghardt, W.; Fromm, W.D.; Geissel, H. The spatial distribution of positron-emitting nuclei generated by relativistic light ion beams in organic matter. Phys. Med. Biol. 1992, 37, 2127-2131. [CrossRef]

6. Bennett, G.; Archambeau, J.; Archambeau, B.; Meltzer, J.; Wingate, C. Visualization and transport of positron emission from proton activation in vivo. Science 1978, 200, 1151-1153. [CrossRef] [PubMed]

7. Min, C.H.; Zhu, X.; Winey, B.A.; Grogg, K.; Testa, M.; El Fakhri, G.; Bortfeld, T.R.; Paganetti, H.; Shih, H.A. Clinical application of in-room positron emission tomography for in vivo treatment monitoring in proton radiation therapy. Int. J. Radiat. Oncol. 2014, 86, 183-189. [CrossRef] [PubMed]

8. Moehrs, S.; Defrise, M.; Belcari, N.; Del Guerra, A.; Bartoli, A.; Fabbri, S.; Zanetti, G. Multi-ray-based system matrix generation for 3D PET reconstruction. Phys. Med. Biol. 2008, 53, 6925-6945. [CrossRef] [PubMed]

9. Nishio, T.; Miyatake, A.; Ogino, T.; Nakagawa, K.; Saijo, N.; Esumi, H. The development and clinical use of a beam on-line PET system mounted on a rotating gantry port in proton therapy. Int. J. Radiat. Oncol. 2010, 76, 277-286. [CrossRef] [PubMed]

10. Oelfke, U.; Lam, G.K.; Atkins, M.S. Proton dose monitoring with PET: Quantitative studies in Lucite. Phys. Med. Biol. 1995, 41, 177-196. [CrossRef]

11. Paganetti, H. Range uncertainties in proton therapy and the role of Monte Carlo simulations. Phys. Med. Biol. 2012, 57, 99-117. [CrossRef] [PubMed]

12. Parodi, K.; Bortfeld, T. A filtering approach based on Gaussian-powerlaw convolutions for local PET verification of proton radiotherapy. Phys. Med. Biol. A 2006, 51, 1991-2009. [CrossRef] [PubMed]

13. Parodi, K.; Crespo, P.; Eickhoff, H.; Haberer, T.; Pawelke, J.; Schardt, D.; Enghardt, W. Random coincidences during in-beam PET measurements at microbunched therapeutic ion beams. Nucl. Instrum. Methods Phys. Res. A 2005, 545, 446-458. [CrossRef]

14. Parodi, K.; Enghardt, W. Potential application of PET in quality assurance of proton therapy. Phys. Med. Biol. 2000, 45, 151-156. [CrossRef] 
15. Dendooven, P.; Buitenhuis, H.J.T.; Diblen, F.; Heeres, P.N.; Biegun, A.K.; Fiedler, F.; van Goethem, M.-J.; van der Graaf, E.R.; Brandenburg, S. Short-lived positron emitters in beam-on PET imaging during proton therapy. Phys. Med. Biol. 2015, 60, 8923-8933. [CrossRef] [PubMed]

16. Sportelli, G.; Belcari, N.; Camarlinghi, N.; Cirrone, G.A.P.; Cuttone, G.; Ferretti, S.; Kraan, A.; Ortuno, J.E.; Romano, F.; Santos, A.; et al. First full-beam PET acquisitions in proton therapy with a modular dual-head dedicated system. Phys. Med. Biol. 2014, 59, 43-60. [CrossRef] [PubMed]

17. Loeffler, J.; Durante, M. Charged particle therapy-optimization, challenges and future directions. Nat. Rev. Clin. Oncol. 2013, 10, 411-424. [CrossRef] [PubMed]

18. Robert, C.; Dedes, G.; Battistoni, G.; Boehlen, T.T.; Buvat, I.; Cerutti, F. Distributions of secondary particles in proton and carbon-ion therapy: A comparison between GATE/Geant4 and FLUKA Monte Carlo codes. Phys. Med. Biol. 2013, 58, 2879. [CrossRef] [PubMed]

19. Torres-Espallardo, I.; Gillam, J.E.; Solevi, P.; Ortega, P.G.; Rohling, H.; Botas, P.; Oliver, J.F.; Llosa, G.; Solaz, C.; Trovato, M.; et al. Optimizing secondary radiation imaging systems for range verification in hadron therapy. In Proceedings of the 2013 IEEE Nuclear Science Symposium and Medical Imaging Conference (2013 NSS/MIC, Seoul, Korea, 27 October-2 November 2013; pp. 1-6.

20. Knopf, A.C.; Parodi, K.; Paganetti, H.; Bortfeld, T.; Daartz, J.; Engelsman, M.; Liebsch, N.; Shih, H. Accuracy of proton beam range verification using post-treatment positron emission tomography/computed tomography as function of treatment site. Int. J. Radiat. Oncol. Biol. Phys. 2011, 79, 297-304. [CrossRef] [PubMed]

21. Tashima, H.; Yamaya, T.; Yoshida, E.; Kinouchi, S.; Watanabe, M.; Tanaka, E. A single-ring OpenPET enabling PET imaging during radiotherapy. Phys. Med. Biol. 2012, 57, 4705-4718. [CrossRef] [PubMed]

22. Zhu, X.; Espana, S.; Daartz, J.; Liebsch, N.; Ouyang, J.; Paganetti, H.; Bortfeld, T.R.; El Fakhri, G. Monitoring proton radiation therapy with in-room PET imaging. Phys. Med. Biol. 2011, 56, 4041-4057. [CrossRef] [PubMed]

23. Crespo, P.; Shakirin, G.; Fiedler, F.; Enghardt, W.; Wagner, A. Direct time-of-flight for quantitative real-time in-beam PET: A concept and feasibility study. Phys. Med. Biol. 2007, 52, 6795-6811. [CrossRef] [PubMed]

24. Crespo, P.; Shakirin, G.; Enghardt, W. On the detector arrangement for in-beam PET for hadron therapy monitoring. Phys. Med. Biol. 2006, 51, 2143-2163. [CrossRef] [PubMed]

25. Parodi, K.; Enghardt, W.; Haberer, T. In-beam PET measurements of beta ${ }^{+}$radioactivity induced by proton beams. Phys. Med. Biol. 2001, 47, 21-36. [CrossRef]

26. Parodi, K. Vision 20/20: Positron emission tomography in radiation therapy planning, delivery, and monitoring. Med. Phys. 2015, 42, 7153-7168. [CrossRef] [PubMed]

27. Paganetti, H.; Jiang, H.; Parodi, K.; Slopsema, R.; Engelsman, M. Clinical implementation of full Monte Carlo dose calculation in proton beam therapy. Phys. Med. Biol. 2008, 53, 4825-4853. [CrossRef] [PubMed]

28. Parodi, K.; Pönish, F.; Enghardt, W. Experimental Study on the Feasibility of In-Beam PET for Accurate Monitoring of Proton Therapy. IEEE Trans. Nucl. Sci. 2005, 52, 778-786. [CrossRef]

29. Amin, T.; Infantino, A.; Lindsay, C.; Barlow, R.; Hoehr, C. Modelling PET radionuclide production in tissue and external targets using Geant4. J. Phys. Conf. Ser. 2017, 874, 012109. [CrossRef]

30. Muraro, S.; Battistoni, G.; Belcari, N.; Bisogni, M.G.; Camarlinghi, N.; Cristoforetti, L.; Del Guerra, A.; Ferrari, A.; Fracchiolla, F.; Morrocchi, M.; et al. Proton therapy treatment monitoring with the DoPET system: Activity range, positron emitters evaluation and comparison with Monte Carlo predictions. JINST 2017, 12, C12026. [CrossRef]

31. Brombal, L.; Barbosa, D.; Belcari, N.; Bisogni, M.G.; Camarlinghi, N.; Cristoforetti, L.; Del Guerra, A.; Fracchiolla, F.; Morrocchi, M.; Sportelli, G.; et al. Proton therapy treatment monitoring with in-beam PET: Investigating space and time activity distributions. Nucl. Instrum. Methods Phys. Res. A 2017, 861, 71-76. [CrossRef]

32. HAMAMATSU. Available online: www.hamamatsu.com (accessed on 15 August2018).

33. SENSL. Available online: www.sensl.com (accessed on 15 August 2018).

34. Haemisch, Y.; Frach, T.; Degenhardt, C.; Thon, A. Fully digital arrays of silicon photomultipliers (dSiPM) a scalable alternative to vacuum photomultiplier tubes (PMT). Phys. Procedia 2010, 37, 1546-1560. [CrossRef]

35. Diblen, F.; Buitenhuis, T.; Solf, T.; Rodrigues, P.; van der Graaf, E.; van Goethem, M.-J.; Brandenburg, S.; Dendooven, P. Radiation hardness of dsipm sensors in a proton therapy radiation environment. IEEE Trans. Nucl. Sci. 2017, 64, 1891-1896. [CrossRef] 
36. Buitenhuis, H.J.T.; Diblen, F.; Brzezinski, K.W.; Brandenburg, S.; Dendooven, P. Beam-on imaging of short-lived positron emitters during proton therapy. Phys. Med. Biol. 2017, 62, 4654-4660. [CrossRef] [PubMed]

37. Ferrero, V. The INSIDE project: In-beam PET scanner system features and characterization. J. Instrum. 2017, 12, C03051. [CrossRef]

38. Piliero, M.A.; Pennazio, F.; Bisogni, M.G.; Camarlinghi, N.; Cerello, P.G.; Del Guerra, A.; Ferrero, V.; Fiorina, E.; Giraudo, G.; Morrocchi, M.; et al. Full-beam performances of a PET detector with synchrotron therapeutic proton beams. Phys. Med. Biol. 2016, 61, N650-N666. [CrossRef] [PubMed]

39. Petzoldt, J.; Roemer, K.E.; Enghardt, W.; Fiedler, F.; Golnik, C.; Hueso-Gonzalez, F.; Helmbrecht, S.; Kormoll, T.; Rohling, H.; Smeets, J.; et al. Characterization of the microbunch time structure of proton pencil beams at a clinical treatment facility. Phys. Med. Biol. 2016, 61, 2432-2440. [CrossRef] [PubMed]

40. Gonzalez, F.H.; Pausch, G.; Petzoldt, J.; Römer, K.E.; Enghardt, W. Prompt Gamma rays detected with a BGO block Compton camera reveal range deviations of therapeutic proton beams. IEEE Trans. Radiat. Plasma Med. Sci. 2017, 1, 76-86. [CrossRef]

41. Schoene, S.; Enghardt, W.; Fiedler, F.; Golnik, C.; Pausch, G.; Rohling, H.; Kormoll, T. An Image Reconstruction Framework and Camera Prototype Aimed for Compton Imaging for In-vivo Dosimetry of Therapeutic Ion Beams. IEEE Trans. Radiat. Plasma Med. Sci. 2017, 1, 96-107. [CrossRef]

42. Yamamoto, S.; Toshito, T.; Komori, M.; Morishita, Y.; Okumura, S.; Yamaguchi, M.; Saito, Y.; Kawachi, N.; Fujimaki, S. Monitoring of positron using high-energy gamma camera for proton therapy. Ann. Nucl. Med. 2015, 29, 268-275. [CrossRef] [PubMed]

43. McCleskey, M.; Kaye, W.; Mackin, D.S.; Beddar, S.; He, Z.; Polf, J.C. Evaluation of a multistage CdZnTe Compton camera for prompt $\gamma$ imaging for proton therapy. Nucl. Instrum. Methods Phys. Res. Sect. A 2015, 785, 163-169. [CrossRef]

44. Rohling, H.; Golnik, C.; Enghardt, W.; Hueso-González, F.; Kormoll, T.; Pausch, G.; Schumann, A.; Fiedler, F. Simulation study of a combined pair production-Compton camera for in-vivo dosimetry during therapeutic proton irradiation. IEEE Trans. Nucl. Sci. 2015, 62, 2023-2030. [CrossRef]

45. Bom, V.; Joulaeizadeh, L.; Beekman, F. Real-time prompt gamma monitoring in spot-scanning proton therapy using imaging through a knife-edge-shaped slit. Phys. Med. Biol. 2012, 57, 297-308. [CrossRef] [PubMed]

46. Smeets, J.; Roellinghoff, F.; Prieels, D.; Stichelbaut, F.; Benilov, A.; Busca, P.; Fiorini, C.; Peloso, R.; Basilavecchia, M.; Frizzi, T.; et al. Prompt gamma imaging with a slit camera for real-time range control in proton therapy. Phys. Med. Biol. 2012, 57, 3371-3405. [CrossRef] [PubMed]

47. Richard, M.-H.; Chevallier, M.; Dauvergne, D.; Freud, N.; Henriquet, P.; Le Foulher, F.; Letang, J.M.; Montarou, G.; Ray, C.; Roellinghoff, F.; et al. Design guidelines for a double scattering Compton camera for prompt- $\gamma$ imaging during ion beam therapy: A Monte Carlo simulation study. IEEE Trans. Nucl. Sci. 2011, 58,87-94. [CrossRef]

48. Golnik, C.; Dersch, U.; Fiedler, F.; Kormoll, T.; Rohling, H.; Enghardt, W. A pair production upgrade for a Compton imager for in-vivo dosimetry at therapeutic proton and ion beams. In Proceedings of the 2011 IEEE Nuclear Science Symposium Conference Record, Valencia, Spain, 23-29 October 2011; pp. 3323-3326.

49. Kormoll, T.; Fiedler, F.; Schöne, S.; Wüstemann, J.; Zuber, K.; Enghardt, W. A Compton imager for in-vivo dosimetry of proton beams-A design study. Nucl. Instrum. Methods Phys. Res. A 2011, 626, 114-119. [CrossRef]

50. Müller, A.; Fiedler, F.; Georg, D.; Hopfgartner, J.; Enghardt, W. 118 in-beam SPECT based in vivo dosimetry: From treatment planning to measured dose. Radiother. Oncol. 2012, 103, S47-S48. [CrossRef]

51. D'Ascenzo, N.; Zhang, X.; Xie, Q. Application of CMOS Technology to Silicon Photomultiplier Sensors. Sensors 2017, 17, 2204-2219. [CrossRef] [PubMed]

52. D'Ascenzo, N.; Brockherde, W.; Dreiner, S.; Schwinger, A.; Schmidt, A.; Xie, Q. Design and Characterization of a Silicon Photomultiplier in 0.35- $\mu \mathrm{m}$ CMOS. J. Electron Dev. Soc. 2018, 6, 74-80.

53. Li, B.; Wang, Y.; Xi, D.; Li, C.; Zeng, C.; Wang, R.; Liang, K.; Yang, R.; Xie, Q.; Han, D. Feasibility Study on Silicon Photomultiplier with Epitaxial Quenching Resistors as the Readout for PET Detectors. IEEE Trans. Nucl. Sci. 2016, 63, 17-21. [CrossRef]

54. Deng, Z.; Xie, Q. Quadratic Programming Time Pickoff Method for Multivoltage Threshold Digitizer in PET. IEEE Trans. Nucl. Sci. 2015, 62, 805-813. [CrossRef] 
55. Deng, Z.; Xie, Q.; Duan, Z.; Xiao, P. Scintillation event energy measurement via a pulse model based iterative deconvolution method. Phys. Med. Biol. 2013, 58, 7815-7827. [CrossRef] [PubMed]

56. Xi, D.; Kao, C.-M.; Liu, W.; Zeng, C.; Liu, X.; Xie, Q. FPGA-only MVT digitizer for TOF PET. IEEE Trans. Nucl. Sci. 2013, 60, 3253-3261. [CrossRef]

57. Wang, X.; Xie, Q.; Chen, Y.; Niu, M. Advantages of digitally sampling scintillation pulses in pileup processing in PET. IEEE Trans. Nucl. Sci. 2012, 59, 498-506. [CrossRef]

58. Xi, D.; Xie, Q.; Zhu, J.; Lin, L.; Niu, M.; Xiao, P.; Chen, C.-T.; Kao, C.-M. Optimization of the SiPM pixel size for a monolithic PET detector. Phys. Proc. 2012, 37, 1497-1503. [CrossRef]

59. Xie, Q.; Kao, C.-M.; Wang, X.; Guo, N.; Zhu, C.; Frisch, H.; Moses, W.W.; Chen, C.T. Potentials of digitally sampling scintillation pulses in timing determination in PET. IEEE Trans. Nucl. Sci. 2009, 56, 2607-2613. [CrossRef] [PubMed]

60. Kim, H.; Kao, C.-M.; Xie, Q.; Frisch, H.; Chen, C.-T.; Tang, F. A multi-threshold method for TOF PET signal processing. Nucl. Instrum. Methods A 2009, 602, 618-621. [CrossRef] [PubMed]

61. Xie, Q.; Kao, C.-M.; Hsiau, Z.; Chen, C.-T. A new approach for pulse processing in Positron Emission Tomography. IEEE Trans. Nucl. Sci. 2005, 52, 988-995.

62. Xi, D.; Liu, X.; Zeng, C.; Liu, W.; Li, Y.; Hua, Y.; Mei, X.; Kim, H.; Xiao, P.; Kao, C.-M.; et al. Modularized compact positron emission tomography detector for rapid system development. J. Med. Imaging 2016, 4, 011006. [CrossRef] [PubMed]

63. Mei, X.; Xi, D.; Zeng, C.; Liu, W.; Liu, X.; Hua, Y.; Kim, H.; Xiao, P.; Kao, C.-M.; Xie, Q. A 72-channel FPGA-only MVT digitizer board and a micro-system for coincidence detection/imaging. In Proceedings of the 2014 IEEE Nuclear Science Symposium and Medical Imaging Conference (NSS/MIC), Seattle, WA, USA, 8-15 November 2014; pp. 1-4.

64. Xi, D.; Zeng, C.; Mei, X.; Wan, L.; Liang, X.; Liu, W.; Liu, X.; Li, B.; Kim, H.; Xiao, P.; et al. A digital PET system based on SiPMs and FPGA-only MVT digitizers. In Proceedings of the 2014 IEEE Nuclear Science Symposium and Medical Imaging Conference (NSS/MIC), Seattle, WA, USA, 8-15 November 2014; pp. 1-3.

65. Li, B.; Xie, Q.; Guo, Y.; Zeng, C.; Wang, S.; Zheng, R.; Wan, L.; Xiao, P. A Panel PET With Window: Design Performance Evaluation and Prototype Development. IEEE Trans. Radiat. Plasma Med. Sci. 2017, 1, 310-321. [CrossRef]

66. Belcari, N.; Attanasi, F.; Moehrs, S.; Rosso, V.; Santos, A.; Spinella, F.; Sportelli, G.; Del Guerra, A. A novel random counts estimation method for PET using a symmetrical delayed window technique and random single event acquisition. In Proceedings of the 2009 IEEE Nuclear Science Symposium Conference Record (NSS/MIC), Orlando, FL, USA, 24 October-1 November 2009; pp. 3611-3614.

67. Beebe-Wang, J.; Vaska, P.; Dilmanian, F.A.; Peggs, S.G.; Schlyer, D.J. Simulation of Proton Therapy Treatment Verificationvia PET Imaging of Induced Positron-Emitters. In Proceedings of the 2003 IEEE Nuclear Science Symposium, Portland, OR, USA, 19-25 October 2003; pp. 2496-2500.

68. Lestand, L.; Montarou, G.; Force, P.; Joly, B.; Lambert, D.; Martin, F.; Rozes, A.; Vert, P.E.; Chadelas, R. In Beam PET Acquisition on $75 \mathrm{MeV}$. text $u^{-1}$ Carbon Beam Using Sampling-Based Read-Out Electronics. IEEE Trans. Radiat. Plasma Med. Sci. 2017, 1, 87-95. [CrossRef]

69. Helmbrecht, S.; Enghardt, W.; Fiedler, F.; Iltzsche, M.; Pausch, G.; Tintori, C.; Kormoll, T. In-beam PET at clinical proton beams with pile-up rejection. Z. Med. Phys. 2017, 27, 202-217. [CrossRef] [PubMed]

70. Binet, S.; Bongrand, A.; Busato, E.; Force, P.; Guicheney, C.; Insa, C.; Lambert, D.; Magne, M.; Martin, F.; Perrin, H.; et al. FPGA-based multichannel data acquisition system for prototype in-beam PET. In Proceedings of the 2013 IEEE Nuclear Science Symposium and Medical Imaging Conference (2013 NSS/MIC), Seoul, Korea, 27 October-2 November 2013; pp. 1-4.

71. Ritt, S.; Dinapoli, R.; Hartmann, U. Application of the DRS chip for fast waveform digitizing. Nucl. Instrum. Methods Phys. Res. A 2010, 623, 486-488. [CrossRef]

72. Joly, B.; Montarou, G.;Lecoq, J.; Bohner, G.; Crouau, M.; Brossard, M.; Vert, P.-E. An optimal filter based algorithm for PET detectors with digital sampling front-end. IEEE Trans. Nucl. Sci. 2010, 57, 63-70. [CrossRef]

73. Varner, G.; Ruckman, L.; Wong, A. The first version buffered large analog bandwidth (BLAB1) ASIC for high luminosity collider and extensive radio neutrino detectors. Nucl. Instrum. Methods Phys. Res. A 2008, 591, 534-545. [CrossRef]

74. Ritt, S. Design and performance of the $6 \mathrm{GHz}$ waveform digitizing chip DRS4.In Proceedings of the 2008 IEEE Nuclear Science Symposium Conference Record, Dresden, Germany, 19-25 October 2008; pp. 1512-1515. 
75. Cambraia Lopes, P.; Bauer, J.; Salomon, A.; Rinaldi, I.; Tabacchini, V.; Tessonnier, T.; Crespo, P.; Parodi, K.; Schaart, D.R. First in situ TOF-PET study using digital photon counters for proton range veri cation. Phys. Med. Biol. 2016, 61, 6203-6230. [CrossRef] [PubMed]

76. (TheCALICE Collaboration). Construction and commissioning of the CALICE analog hadron calorimeter prototype. J. Instrum. 2010, 5, P05004. [CrossRef]

Sample Availability: Samples of the P\&I system are available from the authors.

(C) 2018 by the authors. Licensee MDPI, Basel, Switzerland. This article is an open access article distributed under the terms and conditions of the Creative Commons Attribution (CC BY) license (http://creativecommons.org/licenses/by/4.0/). 